\title{
Geostrophic tripolar vortices in a two-layer fluid: Linear stability and nonlinear evolution of equilibria
}

\author{
J. N. Reinaud, ${ }^{1, a)}$ M. A. Sokolovskiy, ${ }^{2,3, b)}$ and X. Carton ${ }^{4, c)}$ \\ ${ }^{1}$ Mathematical Institute, University of St Andrews, North Haugh, St Andrews KY169SS, United Kingdom \\ ${ }^{2}$ Institute of Water Problems, Russian Academy of Science, 3 Gubkina Street, 119333 Moscow, Russia \\ ${ }^{3}$ P. P. Shirshov Institute of Oceanology, Russian Academy of Science, 36 Nakhimovsky Prospect, \\ 117997 Moscow, Russia \\ ${ }^{4}$ Laboratoire d' Océanographie Physique et Spatiale, IUEM, UBO/UBL, Technopole Brest Iroise, \\ 29280 Plouzane, France
}

(Received 21 September 2016; accepted 6 March 2017; published online 23 March 2017)

\begin{abstract}
We investigate equilibrium solutions for tripolar vortices in a two-layer quasi-geostrophic flow. Two of the vortices are like-signed and lie in one layer. An opposite-signed vortex lies in the other layer. The families of equilibria can be spanned by the distance (called separation) between the two like-signed vortices. Two equilibrium configurations are possible when the opposite-signed vortex lies between the two other vortices. In the first configuration (called ordinary roundabout), the opposite signed vortex is equidistant to the two other vortices. In the second configuration (eccentric roundabouts), the distances are unequal. We determine the equilibria numerically and describe their characteristics for various internal deformation radii. The two branches of equilibria can co-exist and intersect for small deformation radii. Then, the eccentric roundabouts are stable while unstable ordinary roundabouts can be found. Indeed, ordinary roundabouts exist at smaller separations than eccentric roundabouts do, thus inducing stronger vortex interactions. However, for larger deformation radii, eccentric roundabouts can also be unstable. Then, the two branches of equilibria do not cross. The branch of eccentric roundabouts only exists for large separations. Near the end of the branch of eccentric roundabouts (at the smallest separation), one of the like-signed vortices exhibits a sharp inner corner where instabilities can be triggered. Finally, we investigate the nonlinear evolution of a few selected cases of tripoles. Published by AIP Publishing. [http://dx.doi.org/10.1063/1.4978806]
\end{abstract}

\section{INTRODUCTION}

Stationary states for two-dimensional vortices are states in which the vortices do not change shape and ever move forward at a constant velocity or rotate at a constant angular velocity. In the first case, we speak of a translating stationary state, and in the second case, of a rotating stationary state. Finding stationary states for an individual vortex, or for a system of vortices ("V-states" in terminology of Deem and Zabusky ${ }^{1}$ ), is a complex problem which consists in solving nonlinear integral equations.

The classic solution by Kirchhoff ${ }^{2}$ for an elliptical vortex with semi-axes $a$ and $b$ and with vorticity $\omega$ rotating as a solid body at a constant angular velocity $\Omega=\omega a b /(a+b)^{2}$ has been known since 1876. In 1893, Love $^{3}$ showed that this solution is unstable for $\chi=a / b>3$. More recently, Mitchell and Rossi ${ }^{4}$ have given a full analysis of the linear and nonlinear stability of the elliptic vortex as a function of the parameter $\chi$.

The Kirchhoff vortex has many generalisations. In particular, Chaplygin ${ }^{5}$ and later Kida, ${ }^{6}$ Dritschel, ${ }^{7}$ and others showed that the introduction of an external linear velocity field gives rise to pulsating vortices rotating at a time-varying angular velocity associated with a time-varying $\chi$. Polvani and

\footnotetext{
a)Electronic mail: jean.reinaud@st-andrews.ac.uk

b)Electronic mail: sokolovskiy@iwp.ru

c)Electronic mail: xcarton@univ-brest.fr
}

Flierl ${ }^{8}$ introduced the notion of generalised Kirchhoff vortex for a system of $N$ embedded elliptic patches. They studied the linear stability of such solutions.

Kozlov $^{9-11}$ generalised the problem of an elliptic vortex to include the effect of "entrainment" through the introduction of an "effective" bottom friction. This mechanism initiates a cyclone-anticyclone asymmetry that can be seen in the ocean or the atmosphere. Kirchhoff, Chaplygin, and Kida vortices can be recovered as particular cases of Kozlov's solution. ${ }^{9}$

Stationary translating vortex pairs, after the pioneering work of Sadovskii, ${ }^{12}$ have been studied in detail in many papers. ${ }^{1,13-19}$

Other theoretical studies of different translating or rotating stationary states in a barotropic fluid have been the subject of many papers ${ }^{16,17,20-32}$ including the investigations of threeand many-vortex V-states, ${ }^{33-40}$ and asymmetric piecewise uniform vortices. ${ }^{34}$ We draw attention again to the unfortunately little known work by Kozlov, ${ }^{34}$ where the author suggested a general algorithm to construct steady solutions from asymptotic expansions. This algorithm provides the boundaries of steadily translating or rotating $V$-states with high accuracy.

Applications to equilibria in a rotating, stratified fluid, relevant to the oceans and to the atmosphere, have been described in the literature. ${ }^{41-58}$

The present work is performed in the framework of a stratified (two-layer) quasi-geostrophic model and focuses on finite-core analogues of the stationary states of discrete vortex 
systems analysed in previous studies. ${ }^{59-62}$ The main difference between the finite core and the discrete systems consists in the fact that finite core vortices are sensitive to deformation.

Here, we assume that one of the three vortices belongs to one layer and the other two identical vortices are located in a second layer. A complete classification of the relative motion of such a system of three discrete vortices is available in the literature. ${ }^{62}$ Only three types of movements are possible:

(1) Double capture, when all three vortices rotate in the direction determined by the sign of their total intensity, or (in the case of zero total intensity) by the sign of the "strongest" vortex;

(2) simple capture, when a given vortex is under the predominant influence of one of the vortices of the other layer; the second vortex of this layer moves under the action of the combined pair;

(3) a regime where the dominant mechanism is the intralayer interaction between vortices.

Using the terminology used in previous studies, ${ }^{59,62}$ we focus on stationary states which are as follows:

(i) Eccentric roundabouts: Asymmetric collinear constructions of three vortices rotating at a constant angular velocity relative to the vorticity centre, which lies on the line joining all three vortices.

(ii) Tritons: Special cases of eccentric roundabout, when the total intensity of the vortices is equal to zero. Then, the vorticity centre is located at infinity, and the collinear three-vortex structure moves at a constant translation velocity, perpendicular to the line joining the vortices (the regular and chaotic behaviour of discrete tritons has been studied ${ }^{63,64}$ ).

(iii) Ordinary or inverse roundabouts: Symmetric collinear tripolar structures rotating around the centre of vorticity in the direction induced by the central vortex, or in the opposite direction, caused by predominant interlayer interaction of peripheral vortices, correspondingly (the stability of discrete roundabouts is fully described in previous works ${ }^{6-68}$ ).

In the cases (i) and (ii), the configuration satisfies special conditions (or dispersion relations) relating the vortex intensities and the distances separating them. All three types of movements bifurcate from one, degenerate, static collinear state. $^{60}$

Here, we build new stationary states for three vortices with a uniform potential vorticity (PV). Then we analyse the linear stability of the states. Their nonlinear evolution is illustrated on selected examples. Note that the type of motion (iii) was exhaustively studied by Shteinbuch-Fridman et al. ${ }^{56,69}$ for the particular case of zero total potential vorticity. We consider the general case of an arbitrary total vorticity.

It should be noted that configurations of collinear tripoles have been observed in the oceans, for example, with the slope water anticyclonic eddies (swoddies) in the Bay of Biscay. ${ }^{70-72}$ It is therefore important to understand the main characteristics of three-vortex equilibria and the conditions necessary for their existence.

\section{MATHEMATICAL AND NUMERICAL MODELS}

The oceans and the atmosphere can be seen as shallow layers of fluid as the horizontal scales of motion typically far exceed the height of the fluid. In this context, one can model the flow using the shallow-water equations, where the fluid domain is discretised in the vertical direction by layers of fluid of uniform density, each being in hydrostatic balance. In this work, we consider a system of two layers of fluid. Using two layers allows taking into account at the leading order of the effects of the natural density stratification of the oceans. The oceans and the atmosphere are also strongly influenced by the background planetary rotation. In the absence of forcing and dissipation, two-layer flows rotating at the angular velocity $\Omega$ are governed by the equations

$$
\begin{gathered}
u_{j t}+u_{j} u_{j x}+v_{j} u_{j y}-f v_{j}=-\frac{p_{j x}}{\rho_{0}}, \\
v_{j t}+u_{j} v_{j x}+v_{j} v_{j y}+f u_{j}=-\frac{p_{j y}}{\rho_{0}}, \\
p_{j z}=\rho_{j} g, \\
u_{j x}+v_{j y}+w_{j z}=0, \quad j=1,2,
\end{gathered}
$$

where $u_{j}, v_{j}, w_{j}$ are the $j$ th layer velocity vector components along the $x, y, z$-axes, respectively, with the $z$ axis directed downward from the surface; $p$ is pressure; $f=2 \Omega$ is the Coriolis parameter; $\rho_{0}$ is the mean density; $g$ is the acceleration due to gravity. The subscripts $t, x, y, z$ denote partial derivatives with respect to time and to the spatial coordinates, and $\rho_{1}$ and $\rho_{2}\left(\rho_{1} \leq \rho_{2}\right)$ are the constant densities of the incompressible fluids in the upper and lower layers, respectively.

Introducing the vertical component of the relative vorticity in the $j$ th layer $\omega_{j}=v_{j x}-u_{j y}$ and eliminating the pressure from (1) and (2), we obtain

$$
\frac{D_{j}\left(\omega_{j}+f\right)}{D t}-\left(\omega_{j}+f\right) w_{j z}=0, \quad j=1,2,
$$

where $D_{j} a / D t \equiv a_{t}+u_{j} a_{x}+v_{j} a_{y} \equiv a_{t}+J\left(\psi_{j}, a\right)$ is the twodimensional material derivative and $J(b, a)$ the Jacobian, $\psi_{j}$ is the layerwise streamfunction, and $w_{j}$ is the vertical velocity in layer $j$.

For rapid planetary rotation, $\left|\omega_{j}\right| \ll f$, and integrating Equation (5) with respect to $z$ over each layer, taking into account the vertical boundary conditions on the vertical ("rigid lid" on the bottom and top surfaces, pressure continuity at the interface), we obtain

$$
q_{j t}+J\left(\psi_{j}, q_{j}\right)=0, \quad j=1,2,
$$

where

$$
q_{j}=\nabla^{2} \psi_{j}+F_{j}\left(\psi_{3-j}-\psi_{j}\right)+f, \quad j=1,2,
$$

where $q_{j}(x, y)$ is the potential vorticity (PV) in $j$ th layer. The first term in the right hand side of (7) is the relative vorticity of the vortices, the second term is the vorticity associated with the vertical stretching of layerwise columns, and the third term is the background vorticity. The symbol $\nabla^{2}$ is two-dimensional Laplacian and $F_{j}=f^{2} / g^{\prime} h_{j}$ are the layer coupling coefficients. The reduced gravity $g^{\prime}=g \Delta \rho / \rho$ is much smaller than the usual gravity because of the weak relative density difference between layers: $\Delta \rho=\rho_{2}-\rho_{1} \ll \rho_{1}$. The thickness of layer $j$ at rest is $h_{j}$ and $h=h_{1}+h_{2}=$ const. is the fixed total fluid thickness. 
Next, assuming that the PV distribution is piecewiseuniform with constant values $q_{j}^{\alpha}$ inside the finite domains $S_{j}^{\alpha}$ bounded by contours $C_{j}^{\alpha}$, i.e.,

$$
q_{j}(x, y)=\sum_{\alpha=1}^{N_{j}} q_{j}^{\alpha} \Theta\left(S_{j}^{\alpha}\right),
$$

where $N_{1}$ and $N_{2}$ are the numbers of vortex patches in upper and lower layers, respectively, and $\Theta\left(S_{j}^{\alpha}\right)$ is the step function equal to 1 inside and 0 outside the contour $C_{j}^{\alpha}$. $\operatorname{are}^{62}$

Under these assumptions, the streamfunctions in layers

$$
\begin{aligned}
\psi_{j}(x, y)= & h_{j} \sum_{\alpha=1}^{N_{j}} q_{j}^{\alpha} \oint_{C_{j}^{\alpha}} T\left[Q(r)+\frac{h_{3-j}}{h_{j}} Q_{1}(r)\right] d v \\
& +h_{3-j} \sum_{\alpha=1}^{N_{3-j}} q_{3-j}^{\alpha} \oint_{C_{3-j}^{\alpha}} T\left[Q(r)-Q_{1}(r)\right] d v, \quad j=1,2 .
\end{aligned}
$$

Here, $r=\sqrt{\left(x-x^{\prime}\right)^{2}+\left(y-y^{\prime}\right)^{2}}\left(x^{\prime}\right.$ and $y^{\prime}$ are the coordinates of the integrating point), and the notations

$$
\begin{gathered}
T=\frac{\left(x^{\prime}-x\right)\left(\partial y^{\prime} / \partial v\right)-\left(y^{\prime}-y\right)\left(\partial x^{\prime} / \partial v\right)}{r^{2}}, \\
Q=\frac{r^{2}}{4 \pi} \ln r, \quad Q_{1}=\frac{1}{2 \pi \gamma^{2}}\left[\gamma r \mathrm{~K}_{1}(\gamma r)-1\right]
\end{gathered}
$$

are introduced; $v\left(x^{\prime}, y^{\prime}\right)$ is a parametric representation of the contour $C_{j}^{\alpha} ; \mathrm{K}_{1}(a)$ is the modified Bessel function of second kind and first order; $\gamma=L / L_{d}$ ( $L$ is a typical horizontal length scale and $L_{d}=\sqrt{g^{\prime} h_{1} h_{2} / h} / f$ is the Rossby deformation radius).

When modelling the evolution of vortex patches, each uniform $q_{j}^{\alpha}$ vortex is represented by its boundary $C_{j}^{\alpha}$, and its curvilinear coordinate $v$ is discretised by $n_{p}$ nodes. We use here $n_{p}=150$ for high resolution (results have been checked by rerunning a selection of cases at three or six times the resolution with $n_{p}=450$ and $n_{p}=900$. The most accurate results available are shown). Nonlinear simulations are performed using contour surgery ${ }^{73}$ adapted here to the two-layer quasi-geostrophic model.

\section{NUMERICAL SETUP}

We consider tripolar vortices in a two-layer fluid. The general geometry is illustrated in Figure 1. The vortices are uniform patches of potential vorticity $q_{j}^{\alpha}$. The layer depths are set to $h_{1}=h_{2}=h / 2$. This choice is made for simplicity (the sensitivity of the results to other choices will be studied later and is briefly described in Sec. V). Without loss of generality, we set $h=1$ and the mean horizontal radius of the vortices can be set to $1(L=1)$.

The tripolar structure consists of two positive, uniform $\mathrm{PV}$ vortices (referred to as vortices 1 and 3 ) in the upper layer $\left(N_{1}=2\right)$ and of one negative, uniform PV vortex (referred to as vortex 2$)$ in the lower layer $\left(N_{2}=1\right)$. As a consequence, we have $q_{1}^{1} \equiv q_{1}, q_{1}^{2} \equiv q_{3}$, and $q_{2}^{1} \equiv q_{2}$. Initially, the centres of

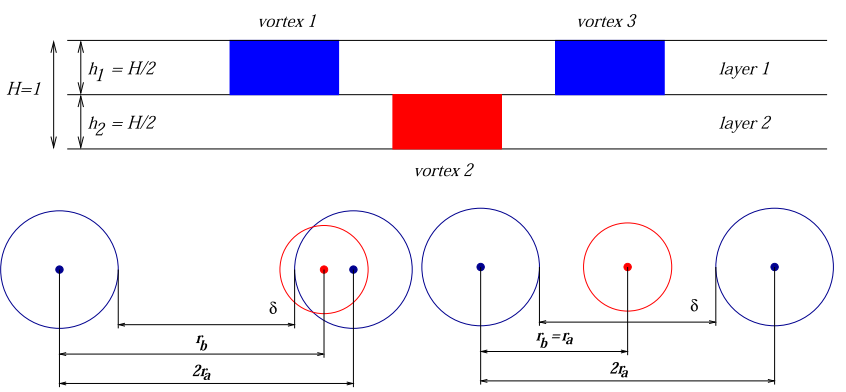

FIG. 1. General geometry of the stationary states for two-layer tripolar vortex structures. Top panel: vertical cross section showing the two layers. Bottom (view form the top): left, asymmetric configuration, generic triton or eccentric roundabout. Right, symmetric configuration, generic ordinary roundabout or inverse roundabout. Hereinafter, the blue (red) circles represent the contours of vortex patches in upper (lower) layer. In this figure only, for simplicity, it is assumed that the vortices have a circular shape.

the three vortices are aligned along the $x$-axis, in a stationary configuration. The vortex structure moves with a constant linear or angular velocity. By convention, vortex 1 will be the vortex on the left and vortex 3 the vortex on the right. The centres of vortices 1 and 3 are separated by a distance $2 r_{a}$. The centres of the upper vortex 1 and the lower vortex 2 are separated horizontally by a distance $r_{b}$. Each vortex with PV $q_{i}$ has the area $A_{i}$ (hence a volume $V_{i}=A_{i} h / 2$ ). We set $q_{1}=q_{3}=q$, taking $q=2 \pi$ without loss of generality. We also set $A_{1}=A_{3}=A=\pi$ (corresponding to $r_{1}=r_{3}$ $=1$ for the equivalent circular vortex). We denote $\rho_{q}=q_{2} / q$ and $\mu=\left(q_{2} A_{2}\right) /(q A)$, such that the total surface-integrated PV is $\Gamma=\left(q_{1} A_{1}+q_{2} A_{2}+q_{3} A_{3}\right) h / 2=\pi^{2}(2+\mu)$. Since $q_{2}<0$, both $\rho_{q}$ and $\mu<0$. It should be noted that $\mu=-2$ corresponds therefore to the case where the overall PV integral is 0 .

When scaling time, we use the simplest model of a pair of opposite signed vortices lying in different layers (an "equivalent" heton $)^{48}$ with a vertical axis $\left(N_{1}=N_{2}=1, r_{b}=0\right.$, $A_{1}=A_{2}=\pi, q_{1}=-q_{2}=q$ ). From (9) we find that the maximum dimensionless azimuthal velocity on both unit circular vortex patches is $V=q \mathrm{I}_{1}(\gamma) \mathrm{K}_{1}(\gamma)$, where $\mathrm{I}_{1}(\gamma)$ is a modified Bessel function of first kind and first order. Suppose now that $\gamma=0.6$ (as in most examples below), then $V \approx 2.57$. Taking as a scale for the horizontal velocity and deformation radius the values $10 \mathrm{~cm} / \mathrm{s}$ and $30 \mathrm{~km}$, respectively, we obtain that the rotation period of fluid particles along the contour is equal to $T \approx 5$ days. We will take this value as the time scale.

For given $\gamma, \mu$, and $\rho_{q}$ a family of steady states can be mapped by the distance $\delta$ between the innermost edges of the lower vortices. This is indicated in Figure 1 where, for the sake of simplicity, we have adopted circular contours to represent the vortices. This distance varies monotonically for the full branches in the range of parameters $\gamma, \mu$ relevant to this study (although it may not be the case outside this range). For given values of $\delta, r, \rho_{q}$, and $\mu$, an equilibrium solution (stationary state or ' $\mathrm{V}$ '-state) is sought. It is reached by an iterative method. The method converges when the boundaries of the vortices match streamlines in the relative reference frame moving with the vortices. ${ }^{14,33,35,49,55,74}$ For tritons $(\mu=-2)$ the reference frame is in uniform translation, while it is in 
uniform rotation in the general case $\mu \neq-2$ (eccentric or ordinary roundabouts). When the equilibrium is reached, $\delta$ is decreased and the calculation is resumed for this new distance. For each state, the iterative method is repeated until the correction to the angular velocity (respectively, translation velocity) is less than a threshold set to $10^{-9}$. The threshold is chosen such that the residual error is smaller than the overall expected accuracy from the discrete representation of the vortices. This has been verified by recomputing a selection of cases with a threshold of $10^{-11}$ (nearer machine precision).

\section{RESULTS}

\section{A. Triton: Translating two-layer V-state, $\mu=-2$}

We start by presenting the results for translating two-layer asymmetric tripolar structures called tritons (item (ii) in the Introduction). For discrete vortices, the triton satisfies ${ }^{62}$

$\frac{r_{b}^{2}-2 r_{a} r_{b}+4 r_{a}^{2}}{2 \gamma r_{a} r_{b}\left(2 r_{a}-r_{b}\right)}-\mathrm{K}_{1}\left(\gamma r_{b}\right)-\mathrm{K}_{1}\left(\gamma\left(2 r_{a}-r_{b}\right)\right)-\mathrm{K}_{1}\left(2 \gamma r_{a}\right)=0$,

and its translation velocity is equal to

$$
v_{t}=\frac{\pi}{2}\left[\frac{2\left(r_{a}-r_{b}\right)}{r_{b}\left(2 r_{a}-r_{b}\right)}-\mathrm{K}_{1}\left(\gamma r_{b}\right)+\mathrm{K}_{1}\left(\gamma\left(2 r_{a}-r_{b}\right)\right)\right] .
$$

For the finite-core vortices, similar relations are obtained numerically. Figure 2 illustrates a generic branch of solutions for $\rho_{q}=-2$ and $\gamma=0.6$. For the sake of completeness, we also provide the results for the equivalent branch of symmetric tripoles (ordinary roundabout). We first plot $r_{b}$ vs $r_{a}$ at equilibrium in Figure 2 (top left). Note that for each value of $r_{a}$, there are two possible values of $r_{b}$, by symmetry. If $r_{b}^{u}$ is the value on the upper part of the branch with a corresponding translation velocity of $v_{t}^{u}$, then, the values for the lower part of the branch denoted by the superscript $l$ are

$$
r_{b}^{l}=2 r_{a}-r_{b}^{u}, \quad v_{t}^{l}=-v_{t}^{u} \equiv v_{t} .
$$

Therefore, only half of the full branch needs to be calculated in practice, and the superscripts $u, l$ will be disregarded. We will use states taken from the upper branch in the paper. The half-branch has two limits. When $r_{a} \rightarrow \infty, r_{b} \sim 2 r_{a}$, as the asymptote drawn in Figure 2 (top left) shows. This limiting case corresponds to a near dipole (or "pseudo-dipole") where two vortices align and are only seen as a single vortex of strength $q A+q_{2} A_{2}=-q A$, from infinity. The second limit is when $r_{a}$ reached its minimum values and corresponds to a turning point for the full branch of solutions. By symmetry, this corresponds to $r_{a}=r_{b}$ and is indicated by the marker $A$ in the figure. Again, by symmetry $v=0$ for this state. Since $r_{a}=r_{b}$, this case also corresponds to a point along the branch for the rotating symmetric roundabout. The branch of tritons bifurcates from the branch of symmetric roundabouts at the point where $v_{t}=\Omega_{p}=0$ (static state). Obviously, the triton is also symmetrical in this limiting state. Ordinary (respectively, inverse) roundabouts lie on the red line to the right (respectively, left) of the markers A. Note that the transition between states with $r_{b} \sim 2 r_{a}$ to the state where $r_{a} \sim r_{b}$ occurs over a rather narrow range of values of $r_{a}$, with indeed a vertical asymptote (turning point) on the branch $r_{b}=f\left(r_{a}\right)$ for the steady states at the point $r_{a}=r_{b}$. This means that the position of the central vortex (vortex 2 ) is very sensitive to changes in $r_{a}$ in this range. In practice, this makes the determination of the branch of solutions by a (linearised) iterative method non-trivial and numerically expensive as any small change in
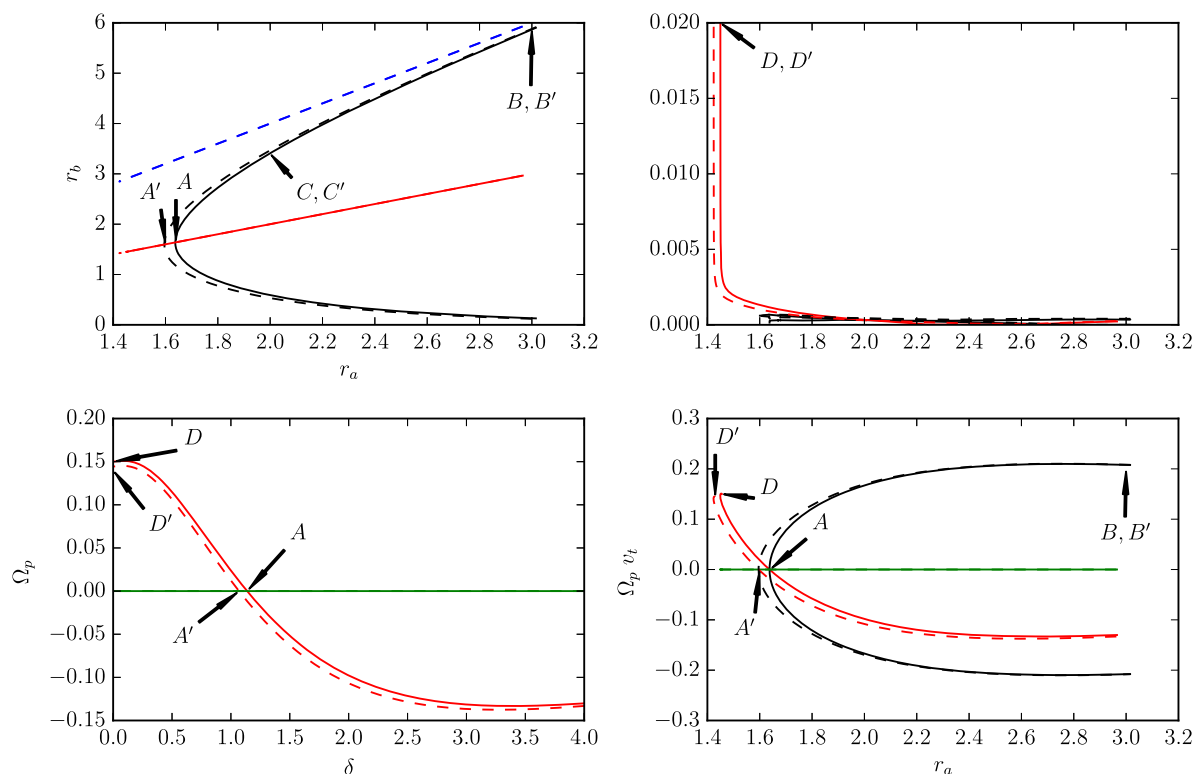

FIG. 2. Characteristics of the equilibria for the case $\mu=-2$, and $\gamma=0.6$ with $\rho_{q}=-2$, i.e., $A_{2}=A$ (solid) and $\rho_{q}=-4$, i.e., $A_{2}=A / 2$ (dashed). Top left: $r_{b}$ vs $r_{a}$ for the triton (black), and symmetric roundabout (reference red line $r_{b}=r_{a}$ ). The dashed blue line indicates the asymptote $r=2 r_{a}$. Top right: growth rate of the two most unstable modes (numerical) calculated. Bottom left: the rotation velocity of the symmetric roundabout vs the gap between the two innermost edges of the side vortices, $\delta$. Bottom right: translation velocity $v_{t}$ for the triton and rotation velocity $\Omega_{p}$ for the symmetric roundabout vs $r_{a}$. Green line indicates the zero level. The markers $A, B, C$, and $D$ indicate the location of tritons and roundabouts illustrated in Figures 3 and 4 for $\rho_{q}=-2$, while the markers $A^{\prime}, B^{\prime}, C^{\prime}$, and $D^{\prime}$ indicate the location of tritons and roundabouts for $\rho_{q}=-4$. 
$\delta$ corresponds to a large displacement of vortex 2 (hence change in $r_{b}$ ). The overall trends are similar to the ones observed in the point vortex calculation ${ }^{62}$ and therefore are explained qualitatively by the analytical solution obtained for the point vortices. But contrary to point vortices, finite-area vortices can split or merge.

Thus, we perform the linear stability analysis of the stationary states, by analysing the deformation modes of the vortices boundary. The method follows straightforwardly that used in three-dimensional quasi-geostrophy, ${ }^{49,55,74}$ adapted here to the two-layer context. All deformations have a time dependence $\propto e^{\sigma t}$, and the algebra leads to an eigenvalue problem for $\sigma=\sigma_{r}+i \sigma_{i}$. The growth rates of instability are given by the real parts $\sigma_{r}$ of the eigenvalues $\sigma$ while $\sigma_{i}$ is their frequencies, see, for example, ${ }^{49,55}$ for full details.

Figure 2 (top right) gives the maximum growth rate obtained numerically along the branch of tritons for the case $\rho_{q}=-2$. The magnitude of the growth rate is on the order of the precision of the computation, which means that the tritons are in fact linearly stable. For the sake of completeness, we also plot the growth rate of the unstable modes for the associated symmetric roundabouts. This configuration is unstable for values of $r_{a}<1.45$, a lower threshold than the minimum $r_{a} \simeq 1.64$ corresponding to the turning point for the tritons. Figure 2 also provides the same information for a different value of $\rho_{q}=-4$, i.e., $A_{2}=A / 2$. The patterns are very similar. We recover the asymptotic behaviour $r_{b} \simeq 2 r_{a}$ for $r_{a} \rightarrow \infty$ corresponding to the limit of a "pseudo-dipole." Finally, we see that this branch of tritons is also neutrally stable, $\sigma_{r} \simeq 0$. It should be noted that the fact that $\sigma_{r}$ is not exactly zero is due to $(i)$ the accuracy of the numerical determination of the steady state by an iterative method resulting in a residual unsteadiness for the states and (ii) the finite accuracy of the calculation in the linear stability analysis.

In the case $\rho_{q}=-2$, Figure 2 (bottom left) shows the rotation velocity for the symmetric roundabouts for the given values of $\mu, \gamma$, and $\rho_{q}$ versus the gap $\delta$ between the innermost edges of the side vortices (vortices 1 and 3 ). Thus we obtained the full branch of equilibrium states, until the two side vortices touch $(\delta=0)$. The state with $\delta=0$ for the branch of symmetric roundabouts is illustrated in Figure 3 (cf. Figure 4c of Ref. 56). The side vortices (vortices 1 and 3 ) exhibit sharp inner corners. The inner edge corresponds to a stagnation point and indicates indeed the end of the branch of solution. Although there are a few differences between the states for $\rho_{q}=-2$ and -4 , the central vortex (vortex 2 ) is slightly more deformed here than for $\rho_{q}=-4$. This is due to the fact that the weaker PV is, the more deformed the vortex becomes in the shear exerted by the other vortices.

The shape of the equilibria for various values of $r_{a}$ is presented in Figure 4. The top frames of Figure 4 correspond to the tritons numerically obtained at the closest of the bifurcation point where the branch intersects the branch of symmetric roundabouts: these are cases labelled $A$ (respectively, $A^{\prime}$ ) in Figure 2 for $\rho_{q}=-2$ (respectively, $\rho_{q}=-4$ ). They correspond to nearly symmetric configurations with $r_{a} \simeq r_{b}$. Note that in this case $r_{a} \simeq 1.6$. Recall that the mean radius of the vortices 1 and 3 is set to unity. This means that the vortices are in fact relatively far apart and are not strongly deformed. This contrasts with the shape of symmetric roundabouts which exist for much smaller values of $r_{a}$, see Figure 3 and further examples. ${ }^{56}$ The second set of frames $\left(B\right.$ and $\left.B^{\prime}\right)$ in Figure 4 corresponds to examples where $r_{a}$ is large enough such that $r_{b} \simeq 2 r_{a}$. Here, vortices 2 and 3 are nearly aligned. Again, the vortices exhibit nearly circular shapes. Indeed, not only are the vortices far apart, thus weakly interacting, but vortex 3 is nearly aligned with vortex 2 . In the absence of vortex 1 , vortices 2 and 3 would exhibit a perfectly circular shape if aligned. Finally, the bottom frames $C$ and $C^{\prime}$ of Figure 4 illustrate tritons for intermediate offset between vortices 2 and 3 . Vortex 3 exhibits the largest deformation while vortices 1 and 2 remain nearly circular. The weak deformation of vortex 1 is again associated with the large separation distance to both vortices 2 and 3 . The asymmetry in deformation between vortices 2 and 3 can be attributed to the PV ratio between these two vortices. Indeed $\left|q_{2} / q_{3}\right|>1$ and vortex 2 is able to withstand a higher level of shear without departing from a nearly circular shape.

Numerical experiments show that the curves presented here are generic of the situation for $\gamma \in[0.2 ; 0.7]$. A summary of the results is provided in Figure 5 for $\rho_{q}=-2, \rho_{q}=-4$, and $\rho_{q}=-1$. The generic trend is that increasing $\gamma$, which increases the vertical coupling, increases the translation velocity and shifts the turning point, where $r_{b}=r_{a}$ to smaller values of $r_{a}$. Note that when $\gamma \rightarrow 0$, the two layers are uncoupled and the lower vortex cannot compensate the mutual rotation induced by the upper vortices, hence the tripolar structure cannot exist as a translating state. When increasing $\gamma$, the ability of the lower (negative) vortex to counteract the rotation of the two upper vortices is enhanced, even when the upper vortices are located close to one another, inducing a strong (positive)
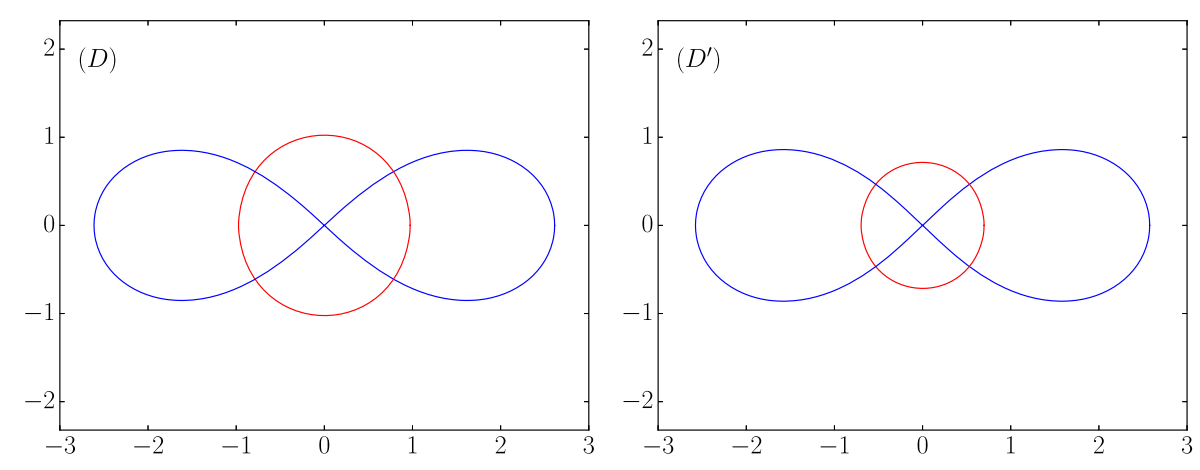

FIG. 3. Top view of the contours of vortex patches of symmetric inverse roundabouts at equilibrium on the plane $(x, y)$ for $\gamma=0.6$ and $\delta=0$. Left: $\rho_{q}=$ -2 ; right: $\rho_{q}=-4$. These states mark ( $D$ and $D^{\prime}$ in Figure 2) the end of the branches of solution as no other state may exist for smaller gaps. 

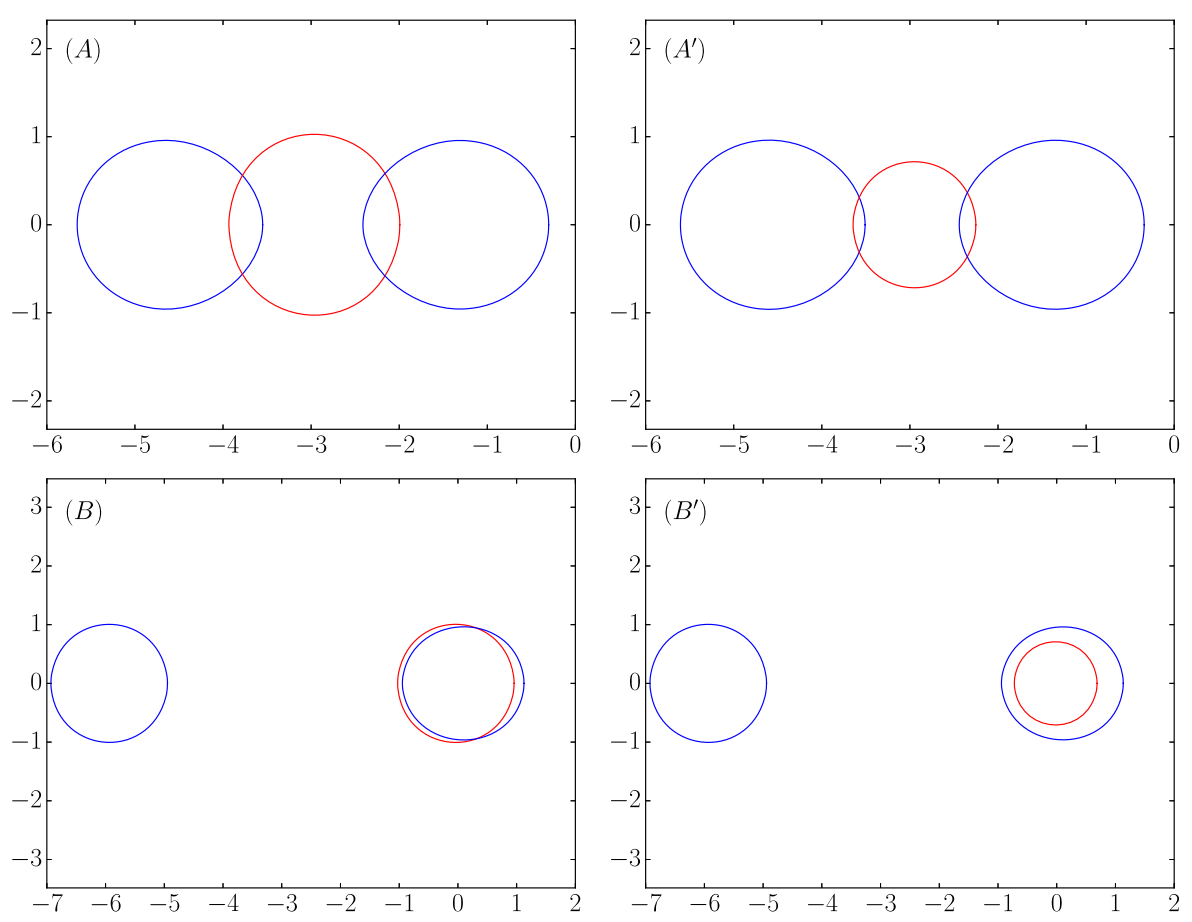

FIG. 4. Top view of the tritons at equilibrium for $\gamma=0.6$. Left, $\rho_{q}=-2$ in vicinity of $(A): r_{a}=1.64 \simeq r_{b}=1.65$; in $(B): r_{a}=3$, and $r_{b}=5.9$; and in (C): $r_{a}=2, r_{b}=3.4$. Right, $\rho_{q}=-4$ in vicinity of $\left(A^{\prime}\right): r_{a}=1.6, r_{b}=1.62$; in $\left(B^{\prime}\right): r_{a}=3, r_{b}=5.9$; and in $\left(C^{\prime}\right): r_{a}$ $=2, r_{b}=3.47$. The frame labels are the markers in Figure 2.
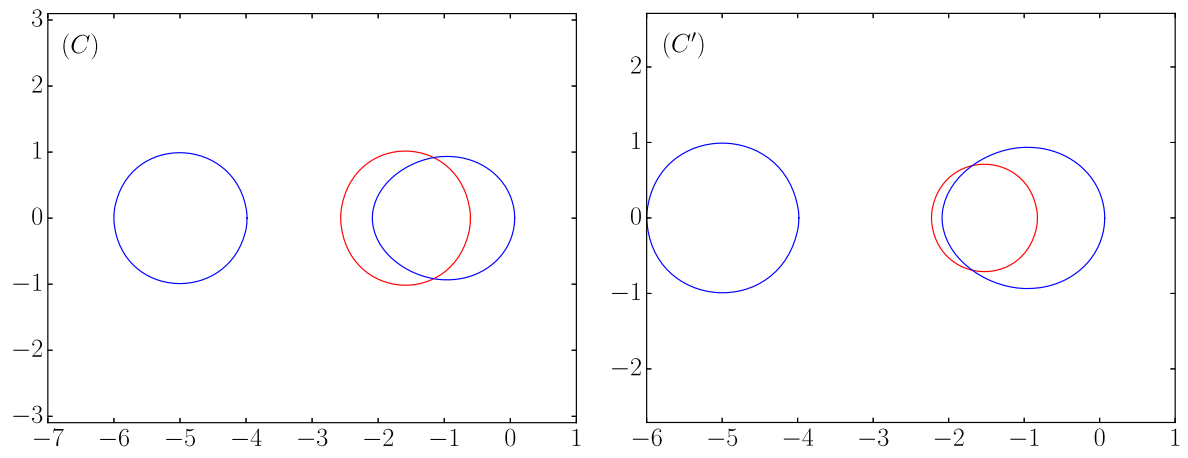

rotation. Therefore, the triton can exist over a larger range of values for $r_{a}$ as $\gamma$ is (moderately) increased. We will next see what happens if $\gamma$ exceeds a threshold depending on $\rho_{q}$ (but typically $\geq 0.8$ ). Finally, in the range $\gamma \in[0.2 ; 0.7]$, the translating, asymmetric tritons are linearly, neutrally stable.

The qualitative dependence of the parameters $r_{b}$ and $v_{t}$ vs $r_{a}$ for the steady states is the same for different values of $\rho_{q}$. We see that the quantitative dependence of the parameters $r_{b}$ vs $r_{a}$ and $v_{t}$ vs $r_{a}$ is small. This is due to the fact that these parameters are, at the leading order at least, dictated by the strength of the vortices $q_{i} V_{i}$ rather than by the individual values of the PV $q_{i}$ and associated area $A_{i}$. However, the actual shape of the vortices is strongly influenced by the PV ratio. As mentioned before, the ability of a vortex to withstand external shear with little deformation is related to its strong PV. Note that the curves in Figure 5 are qualitatively similar to the curves provided by formulas (10) and (11) for the discrete tritons (Figure 2.64 of Ref. 62).
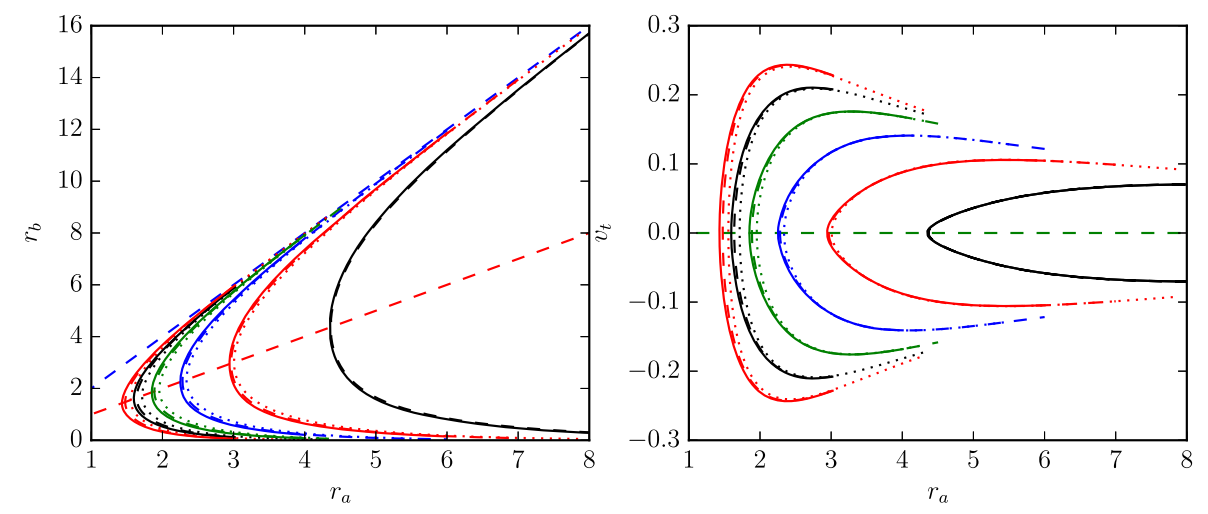

FIG. 5. Characteristics of the equilibria for $\mu=-2$ (tritons): Left, $r_{b}$ vs $r_{a}$ and right, $v_{t}$ vs $r_{a}$. Curves are organised as follows: $\rho_{q}=-1$ (dotted lines), -2 (dashed lines), and -4 (solid lines). Then, from left to right $\gamma=0.7$ (red), 0.6 (black), 0.5 (green), 0.4 (blue), 0.3 (red), 0.2 (black). 

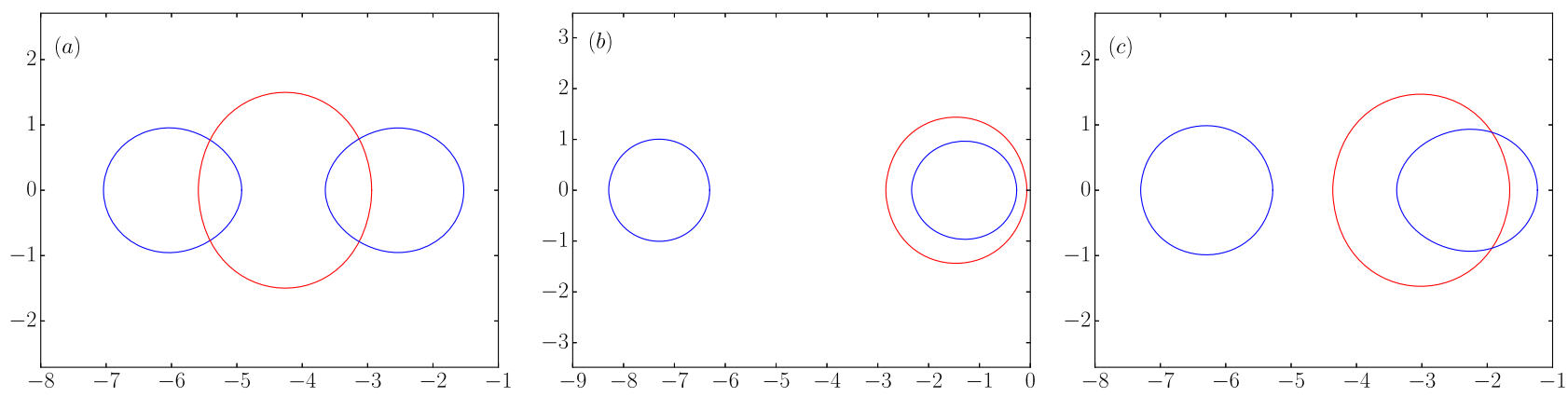

FIG. 6. Top view of the triton at equilibrium for $\gamma=0.6$ and $\rho_{q}=-1$ with $(a): r_{a}=1.71, r_{b}=1.73,(b): r_{a}=3, r_{b}=5.89$, and $(c): r_{a}=2, r_{b}=3.28$.

Figure 6 illustrates the shape of vortices for $\rho_{q}=-1$ (when $A_{2}=2 A$ ), for $r_{a} \simeq r_{b}, r_{a} \simeq 2 r_{b}$, and an intermediate case. Compared with the shapes of the steady states for $\rho_{q}=-2,-4$ (see Figure 4), it is clear that vortex 2 is more deformed as its ability to withstand shear is lowered. This helps understand the weak dependence of the values of $r_{b}$ and $v_{t}$ from a given value of $r_{a}$ for the steady states, depending on $\rho_{q}$. The deformation of vortex 2 weakens its influence. This affects the interaction in a similar (albeit much weaker) way as changing $\gamma$ as discussed previously.

When increasing $\gamma$ further, the situation changes. We cannot reach the point where the branch would intersect the branch of ordinary/inverse roundabouts at $r_{a}=r_{b}$. In fact, we cannot obtain equilibria for $r_{a}$ smaller than a threshold value. This value of $r_{a}$ still corresponds to a situation for which $r_{b}>r_{a}$. Moreover, when decreasing further the gap $\delta$, the corresponding value of $r_{a}$ for the new steady states increases. There is a new turning point in the branch of solutions. We illustrate this for $\rho_{q}=-4$ and $\gamma=0.8$. This case is generic and similar behaviours are obtained for $\rho_{q}=-2$ (not shown). The turning point in the plane $\left(r_{a}, r_{b}\right)$ is indicated in Figure 7 (top left).

The shape of the tritons around the turning point, indicated in Figure 7 by the markers $E$ and $F$, is illustrated in Figure 8 . We could not continue numerically the branch for smaller values of $\delta$ (i.e., beyond the marker $F$ ). Other numerical experiments however indicate that the branch could continue until vortex 3 exhibits a sharp inner edge, which marks the physical end of the branch of solution. Recall that since the vortex boundary coincides with a streamline, a sharp corner indicates the presence of a stagnation point $(u, v)=(0,0)$. A brief analysis of the deformation of the vortex is proposed in the Appendix. To better understand why the branch does not reach a state where $r_{b}=r_{a}$, we look at the corresponding branch for the symmetric roundabouts. We observe in Figure 7 (bottom left) that, contrarily to the cases with $\gamma \in[0.2 ; 0.7]$, the rotation rate for the ordinary roundabout is always dominated by the (negative) rotation of the central negative PV vortex. Even when $\delta=0$, and the side vortices are at their closest, the positive rotation they induce cannot counteract the negative rotation. There is no point with $\Omega_{p}=0$ on this branch where the branch of tritons would bifurcate from. This regime cannot be captured by a discrete system of point vortices and is specific to the more realistic finite core vortex system.

We next perform the linear stability analysis for the branch of tritons. Unstable modes are found. The first two modes, of equal growth rate $\sigma_{r}$, arise at $r_{a} \simeq 2.25$. Their emergence is indicated by the marker $G$ in Figure 7 . The growth rate associated with these two modes first increases as $r_{a}$ is decreased to reach a peak $\sigma_{r} \simeq 0.079$ at $r_{a} \simeq 2.02($ marker $H$ ), then decreases for small $r_{a}$. The mode is stable for $r_{a}<r_{a} \simeq 1.95$
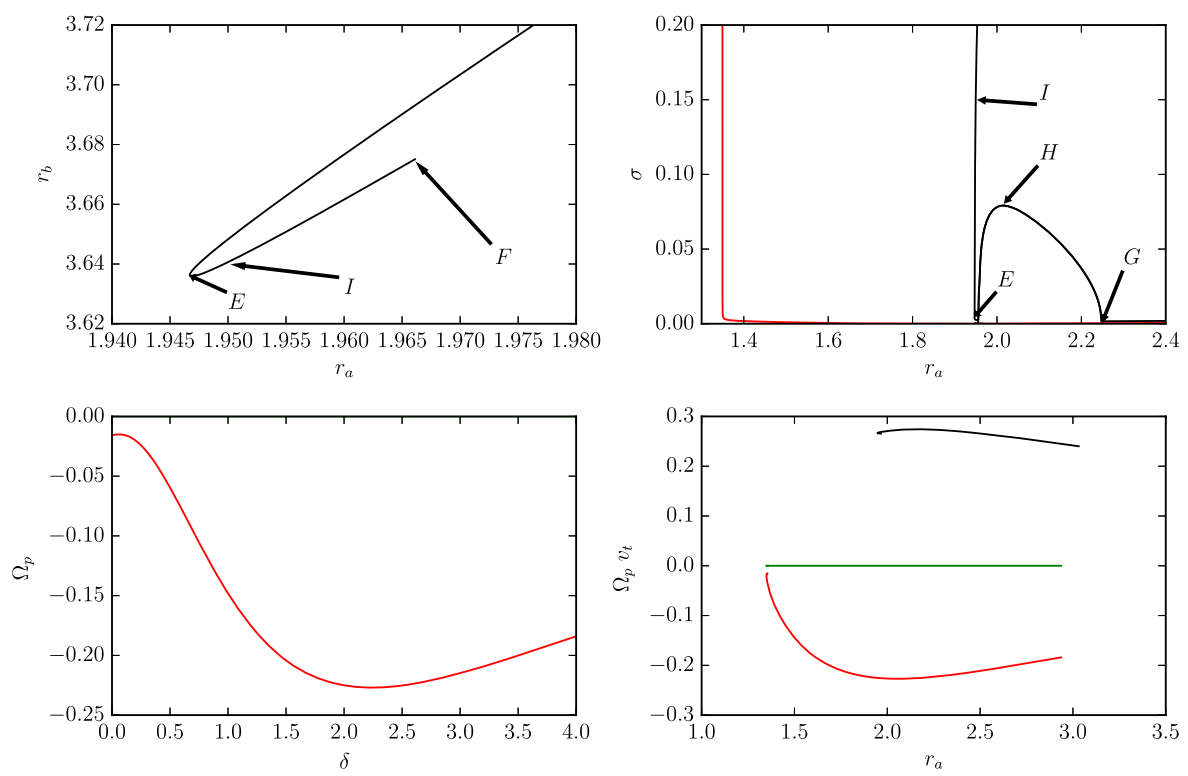

FIG. 7. Characteristics of the equilibria with $\mu=-2$ (tritons) for $\rho_{q}=-4$ and $\gamma=0.8$, incomplete branch. Top left, $r_{b}$ vs $r_{a}$. Top right, growth rate of the two most unstable modes (numerical) calculated for tritons and symmetric roundabouts. Bottom left: rotation velocity of the symmetric ordinary roundabouts vs the gap between the two innermost edges of the side vortices, $\delta$. Bottom right: translation velocity $v_{t}>0$ for the tritons (upper curve), and rotation $\Omega_{p}<0$ (lower curve) for the symmetric ordinary roundabouts vs $r_{a}$. The central line offers a reference at $\Omega_{p}=v_{t}=0$. 

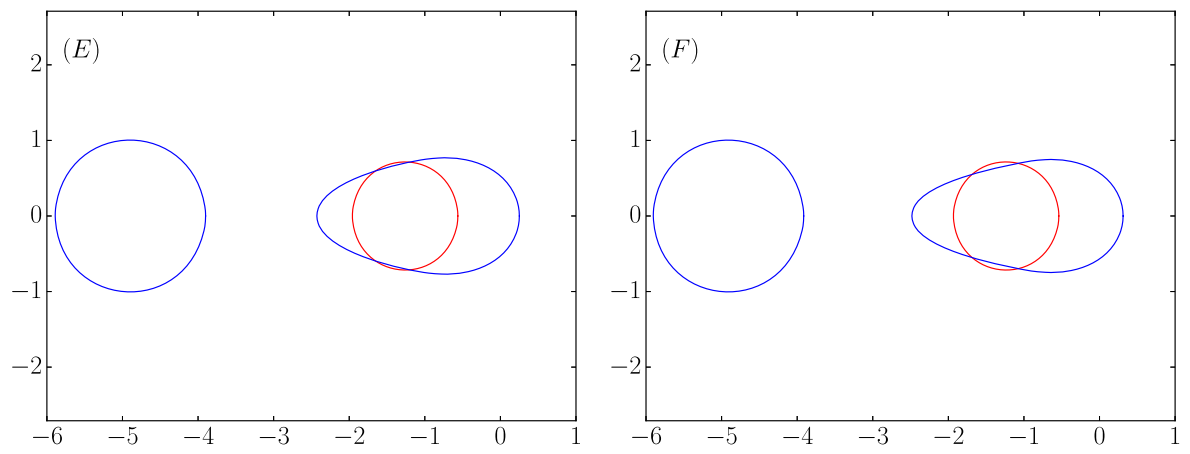

FIG. 8. Top view of the vortices at equilibrium for $\mu=-2$ (tritons), for $\rho_{q}=$ -4 and $\gamma=0.8$, corresponding to the markers $E$ (left) and $F$ (right) in Figure 7. (marker $E$ ), but a new mode of instability emerges there. The former mode is relatively weak while the latter has a growth rate which increases significantly as $r_{a}$ is further decreased (see, for example, marker $I$ ). The vortices for cases $E$ and $F$ are illustrated in Figure 8.

The eigenvector of the modes of instability allows reconstructing its spatial structure (deformation of the vortex boundary). They are illustrated in Figure 9 for cases $H$ and $I$. The vortex which mostly undergoes instability in the nonlinear regime is the most deformed one, vortex 3 . The deformation mode is dominated by the azimuthal wave number $m=2$. This mode corresponds to the elongation of the vortex in a direction (and conversely its thinning in the near perpendicular direction by area conservation).

The instability is illustrated by performing a nonlinear simulation of the equilibrium. There is no forcing imposed and the perturbations arise from the numerical noise associated with the finite resolution of the vortices. We illustrate the case corresponding to peak instability for the first unstable modes, with $r_{a} \simeq 2.02$ (marker $H$ of Figure 7 ) in Figure 10 . The larger, upper vortex (vortex 3) lying over the smaller yet more intense, lower vortex (vortex 2) destabilises on an azimuthal mode $m=2$ and elongates. A filament, generated at its sharper inner edge, spreads and wraps around vortex 1 which is in the same layer. For $r_{a}=1.95$ with $\sigma_{r} \simeq 0.15$, the same scenario repeats. The flow evolution (not shown) is dominated by the destabilisation of vortex 3 according to an instability dominated by an azimuthal wave number $m=2$, in agreement with the linear stability analysis. Again, a filament is generated at the acute inner corner of vortex 3 after a preliminary phase of elongation towards vortex 1 . This instability appears to be generic of the configurations where vortex 3 is more deformed than any of the two other vortices, in particular near its inner edge.

\section{B. Common case $\mu \neq-2$ : Eccentric roundabout (rotating asymmetric two-layer tripolar V-state)}

The dispersion equation for point vortex eccentric roundabout (item (i) in the Introduction) is ${ }^{62}$

$$
\frac{1}{2 \gamma r_{a}}+\frac{2 r_{a}(1+\mu)}{\gamma r_{b}\left(2 r_{a}-r_{b}\right)}+\mathrm{K}_{1}\left(2 \gamma r_{a}\right)+\frac{\left(2 r_{a}+\mu r_{b}\right) \mathrm{K}_{1}\left(\gamma\left(2 r_{a}-r_{b}\right)\right)-\left(2 r_{a}(1+\mu)-r_{b} \mu\right) \mathrm{K}_{1}\left(\gamma r_{b}\right)}{2\left(r_{a}-r_{b}\right)}=0
$$

For a collinear configuration of three discrete vortices located along the $x$-axis with the vortex of the lower layer at $(0,0)$, satisfying the above equations (eccentric roundabout), the solid body rotating at angular velocity $\Omega_{p}$ relative
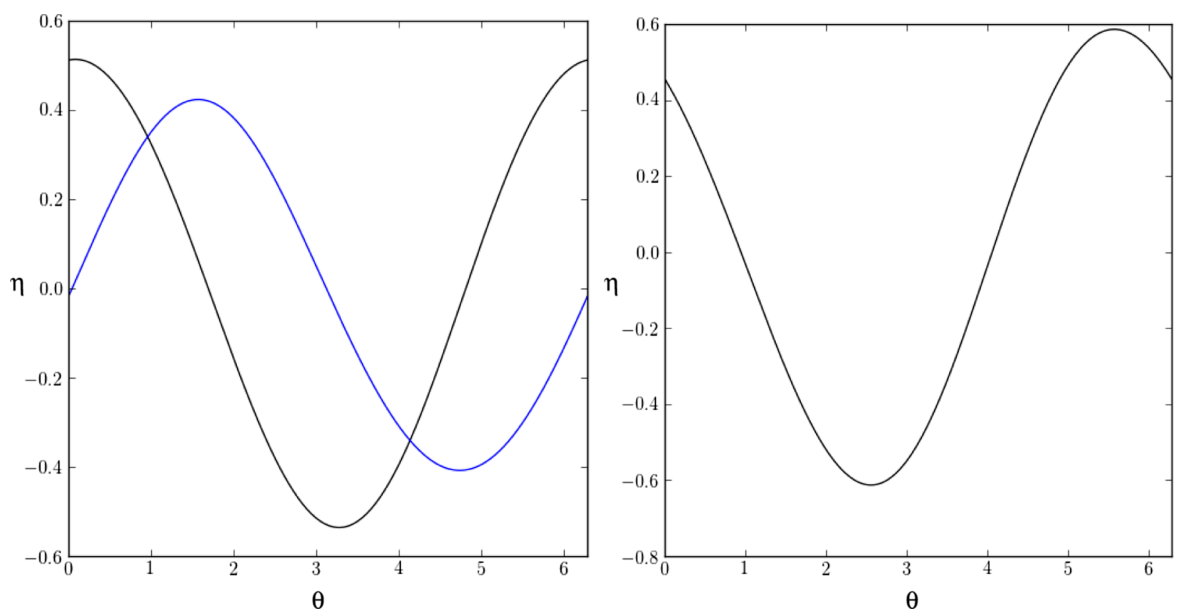

FIG. 9. Spatial structure of the two most unstable modes for vortex 3 , the lower (unstable) pole of the triton for $\rho_{q}=-4$ and $\gamma=0.8$. Left, marker $H$ in Figure 7 with $r_{a} \simeq 2.1$. There are unstable two modes of equal growth rates. Right: marker $I$ in Figure 7 with $r_{a} \simeq$ 1.95 (there is only one unstable mode in this case). The horizontal axis represents the azimuthal angle $\theta$ along the contour. The vertical axis corresponds to the amplitude $\eta$ of deformation mode (eigenvector) of the contour (departure from equilibrium). 

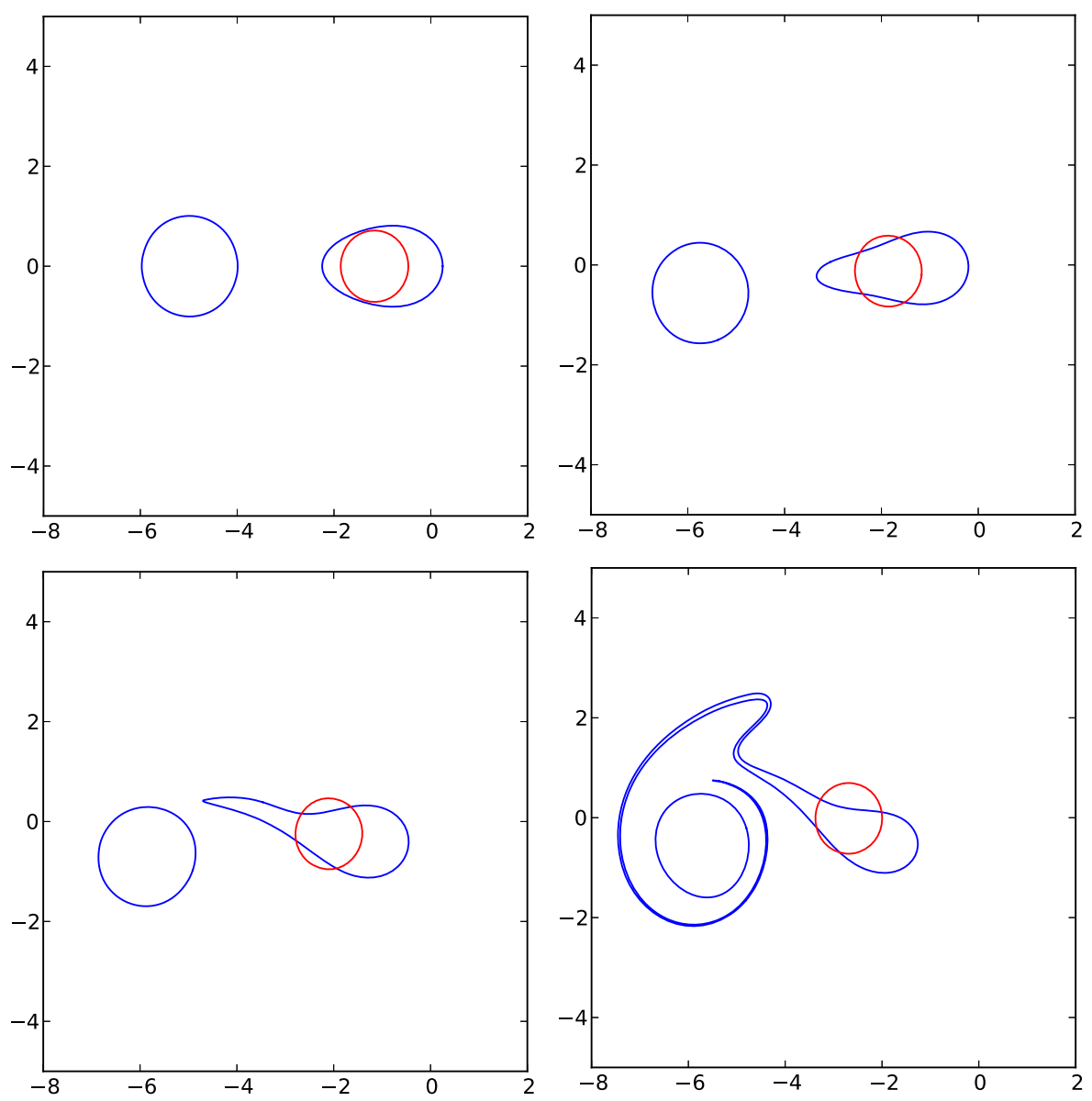

FIG. 10. Time evolution of the vortex patches for an unstable equilibrium with $\mu=-2$ (triton) for $\rho_{q}=-4$ and $\gamma=0.8$. The equilibria correspond to the marker $H$ in Figure 7 with $r_{a} \simeq 2.02$. Frames displayed correspond to $t=0,79,84,89$. The frames are plotted in the reference frame rotating with the equilibrium. the vorticity centre $\left(x_{c}, y_{c}\right)$ is given by

$$
\left(x_{c}, y_{c}\right)=\left(\frac{2\left(r_{a}-r_{b}\right)}{\mu+2}, 0\right)
$$

with

$$
\Omega_{p}=\frac{(\mu+2) \pi}{2\left(2 r_{a}+r_{b} \mu\right)}\left(\frac{r_{b}+2 r_{a} \mu}{2 \gamma r_{a} r_{b}}-\mu \mathrm{K}_{1}\left(\gamma r_{b}\right)+\mathrm{K}_{1}\left(2 \gamma r_{a}\right)\right) .
$$

When $\mu \rightarrow-2$, the dispersion equation (13) gives (9), $x_{c} \rightarrow \infty$ and $\Omega_{p} \rightarrow 0$ and we recover the case of a triton. For $\mu \neq-2$ however, $x_{c}$ is finite. Similarly, for finite core vortices with $\mu \neq-2$, the centre of vorticity is no longer rejected to infinity, and an eccentric roundabout no longer translates as the tritons did, but rotates.

We illustrate the asymmetric two-layer tripoles for 3 values of the parameter $\mu$, the strength ratio between the lower vortex (vortex 2) and one of the two upper vortices (vortex 1 or 3): $\mu=-1.2,-1.5$, and -1.8 . We first determine $r_{b}$ vs $r_{a}$ and the angular velocity $\Omega_{p}$ (for given $r_{a}$ ) for the steady asymmetric tripoles (eccentric roundabout). The numerical procedure is the same as for the triton, except that the equilibria are sought in a rotating frame. We start with $r_{a} \gg 1$ and $r_{b} \simeq 2 r_{a}$. When equilibrium is reached, the distance between the side vortices (vortices 1 and 3 ) is reduced and the procedure is resumed. For tritons, there is no steadily translating state below some $r_{a \min }$. The numerical procedure used to obtain the stationary states stops at this minimum, as no equilibrium exists below this threshold. Recall that the state corresponding to $r_{a \text { min }}$ is a symmetric state and is the point of intersection with the branch of symmetric tripoles (ordinary/inverse roundabouts). For asymmetric tripoles $(\mu \neq-2)$, since both branches correspond to rotating states, the procedure started along the branch of eccentric roundabouts naturally follows the branch of symmetric tripoles for values of $r_{a}<r_{a \text { min }}$. Obtaining stationary states for $r_{a} \simeq r_{a \text { min }}$ is however difficult numerically due to the proximity of multiple solutions for the equilibria. On the other hand, the full branch of symmetric states can also be browsed by imposing a double symmetry (with respect to the $x$ and $y$ axes).

Figure 11 shows $r_{b}$ vs $r_{a}$ and $\Omega_{p}$ vs $r_{a}$ for $\rho_{q}=-2$ and -4 , respectively, and $\mu=-1.2,-1.5$, and -1.8 . The sharp turning point in the curves $\Omega_{p}$ vs $r_{a}$ corresponds to the point when $r_{a}$ reaches $r_{b}$; this point is the intersection between the branches of symmetric and eccentric roundabouts. Note that the kink in the curve appears at $\Omega_{p} \simeq 0$. In theory, the kink should correspond to $\Omega_{p}=0$. We cannot reach this state exactly in practice (due to the accuracy of the solution near the intersection between the two branches of solutions). This difficulty is related to the sharpness of the kink (vertical tangent in $\Omega_{p}$ vs $r_{a}$ ) at this point. The graph $r_{b}$ vs $r_{a}$ presents two distinct parts. The linear part of the graph for small values of $r_{a}$ corresponds to the branch of symmetric states (where by construction $r_{a}$ $=r_{b}$ ) while the curved part for larger $r_{a}$ corresponds to the eccentric roundabouts. This part asymptotically tends to $r_{b}$ $\simeq 2 r_{a}$ for $r_{a} \rightarrow \infty$. The abrupt change of dependence of $\Omega_{p}$ 

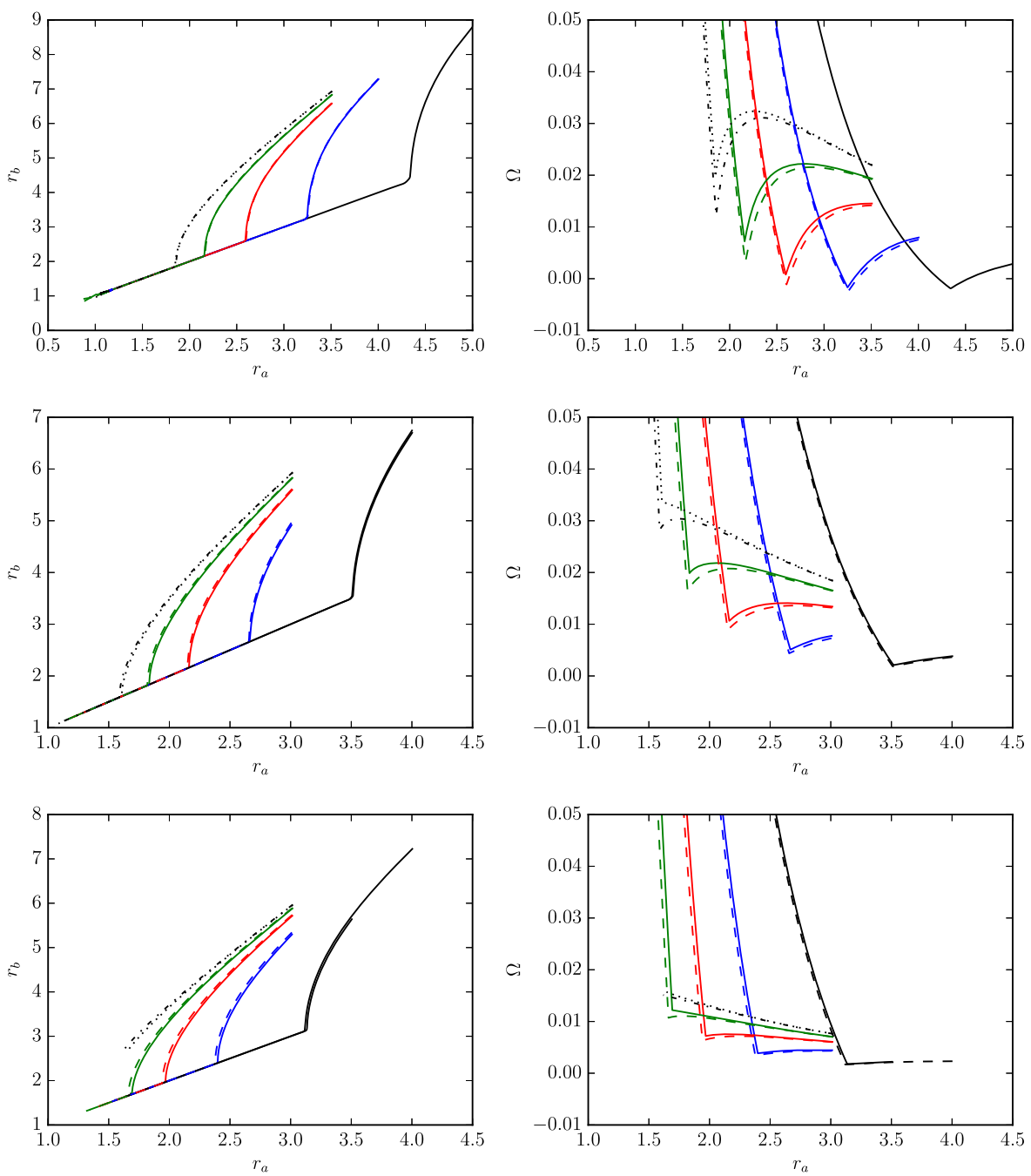

FIG. 11. Characteristics of eccentric roundabouts at equilibrium for $\mu=$ -1.2 (top panels), -1.5 (middle panels), -1.8 (bottom panels), and $\left(\rho_{q}, \gamma\right)=$ $(-2,0.3)$ solid black, $(-2,0.4)$ solid blue, $(-2,0.5)$ solid red, $(-2,0.6)$ solid green, $(-2,0.7)$ dotted black, $(-4,0.3)$ dashed black, $(-4,0.4)$ dashed blue, $(-4,0.5)$ dashed red, $(-4,0.6)$ dashed green, $(-4,0.7)$ dotted-dashed black. Left graphs $r_{b}$ vs $r_{a}$ at equilibrium. Right graphs $\Omega_{a}$ vs $r_{a}$. Note that the kink in the curves $\Omega_{p}$ vs $r_{a}$ should occur (in theory) at $\Omega_{p}=0$ exactly. on $r_{a}$ was already observed in the point vortex calculation and therefore can be explained by a simple analytical calculation. ${ }^{62}$ The branches corresponding to the asymmetric tripoles are qualitatively very similar to the ones for the tritons $(\mu=-2)$. Again, we recover a strong influence of $\gamma$. Increasing $\gamma$ lowers the values of $r_{a m i n}$ and overall increases (for a given $r_{a}$ ) $\Omega_{p}$. Both trends are related to the increased coupling between the layers, as explained in the case $\mu=-2$. As in the case of the tritons, and for the same reasons, the branches of solution cannot reach the symmetric states for large values of $\gamma$ (not further detailed here).

On the other hand, by changing $\mu$, we change the relative importance of the lower, negative vortex on the pair of corotating upper, positive vortices. Lowering the influence of the negative vortex makes, overall, the rotating rate $\Omega_{p}$ reach higher, positive values. It also shifts $r_{a \min }$ to higher values.
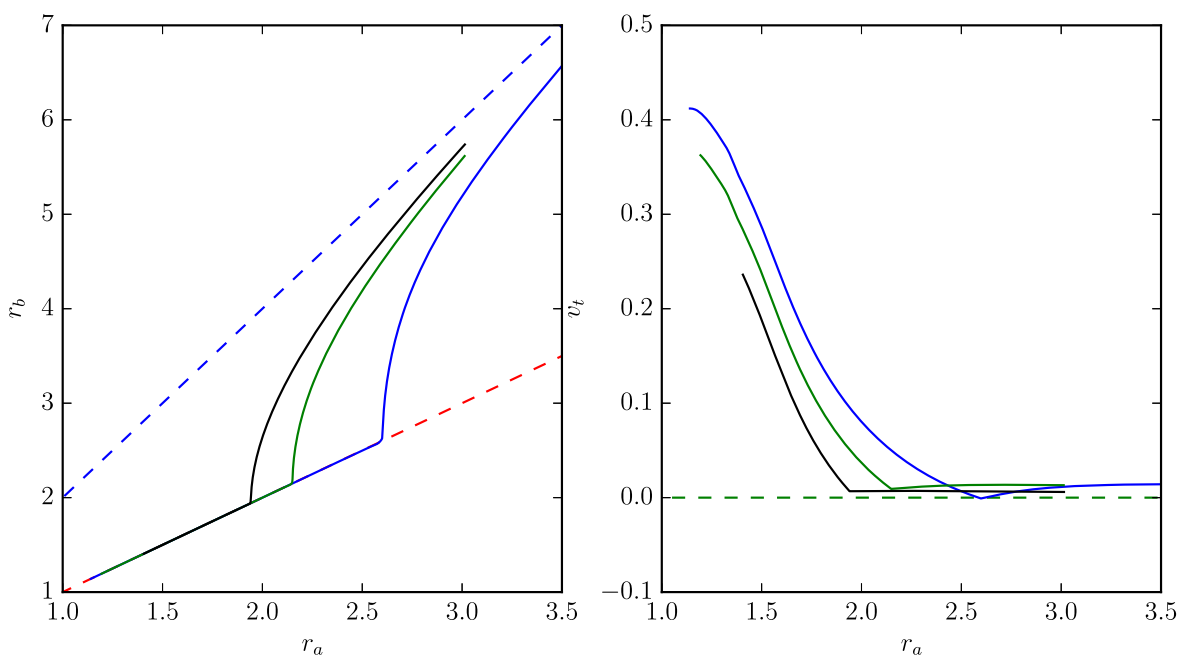

FIG. 12. Comparison between 3 branches of eccentric roundabouts at equilibrium with $\rho_{q}=-4, \gamma=0.5$, and $\mu=-1.2$ (solid blue), -1.5 (solid green), and -1.8 (solid black). Left: $r_{b}$ vs $r_{a}$, and right: $\Omega_{p}$ vs $r_{a}$. 


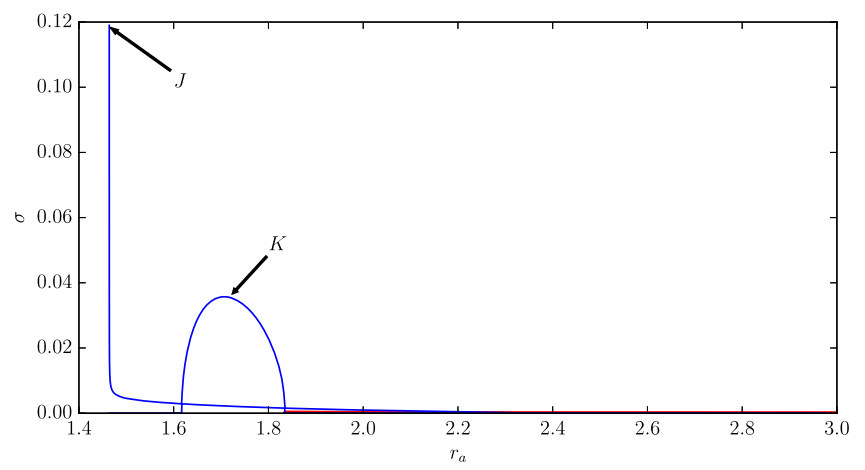

FIG. 13. Growth rates of the two most unstable modes vs $r_{a}$ for the equilibria with $\rho_{q}=-2, \mu=-1.5, \gamma=0.6$ (bottom) for eccentric roundabout (red) and ordinary/inverse roundabouts (blue).

Again, this is related to the reduced ability of the lower vortex to adapt to the positive rotation induced by the upper vortices. This influence is illustrated in Figure 12. We also recover that the influence of $\rho_{q}$ on the global parameters such as $r_{b}=f\left(r_{a}\right)$ and $\Omega_{p}=g\left(r_{a}\right)$ (for equilibrium) is moderate.

Next, we present the linear stability of the full branches of asymmetric tripoles and the corresponding branch of symmetric tripoles. Figure 13 shows the growth rates of the two most unstable modes for $\mu=-1.5, \rho_{q}=-2$, and $\gamma=0.6$. Both cases are qualitatively very similar. The branch corresponding to the eccentric roundabout (asymmetric case) represented in red shows that the configurations are neutrally stable (within the accuracy of the calculation). This branch stops at $r_{a}=1.835$ for $\gamma=0.6$.

For lower values of $r_{a}$, the only possible configuration is the symmetric one. In both cases, the intersection between the branches of eccentric and ordinary/inverse roundabouts is the starting point of an unstable mode along the symmetric branch. The growth rate of this mode first increases as $r_{a}$ is decreased, to reach a maximum $\sigma=0.0357$ at $r_{a}=1.71$ for $\gamma=0.6$ (with $q=2 \pi$ ). Note that the amplitude of the maximum growth rate remains small $\left(O\left(10^{-2}\right)\right)$. The growth rate then decreases for decreasing $r_{a}$ and the mode disappears for $r_{a}=1.62$. The second mode appears and rapidly grows for $r_{a}<1.47$.

We illustrate the unstable regime by performing nonlinear simulations of $\mu=-1.5, \rho_{q}=-2$, and $\gamma=0.6$. The instabilities are not forced and arise from the amplification of small numerical errors. We first investigate the nonlinear evolution of a strongly unstable state with $r_{a}=1.46$ (indicated by the marker $J$ in Figure 13). Results are presented in Figure 14. It should be noted that the results are presented in the reference frame rotating with the equilibrium. At $t=0$, the two positive vortices of the upper layer at equilibrium exhibit highly deformed inner edges. Their shape resembles that of two co-rotating vortices at equilibrium close to the limit of vortex merger. The instability that develops is indeed related to the merger of the two upper, like-signed vortices. The two
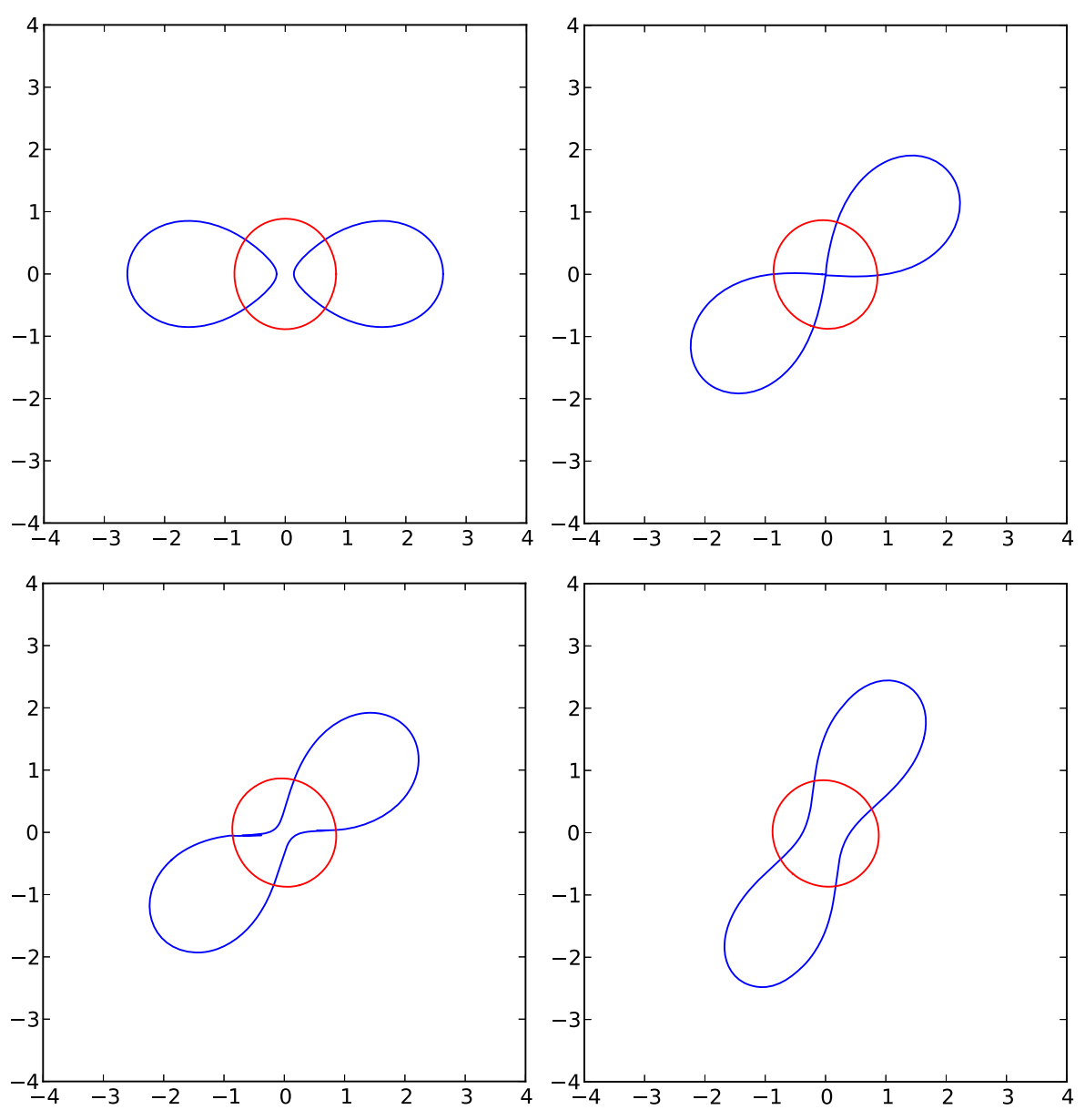

FIG. 14. Top view of the unstable symmetric inverse roundabout at $t=0,29$, 30 , and 39 for $\rho_{q}=-2, \mu=-1.5$, $\gamma=0.6$, and $r_{a}=1.46$, see marker $J$ in Figure 13. The frames are plotted in the reference frame rotating with the equilibrium. 

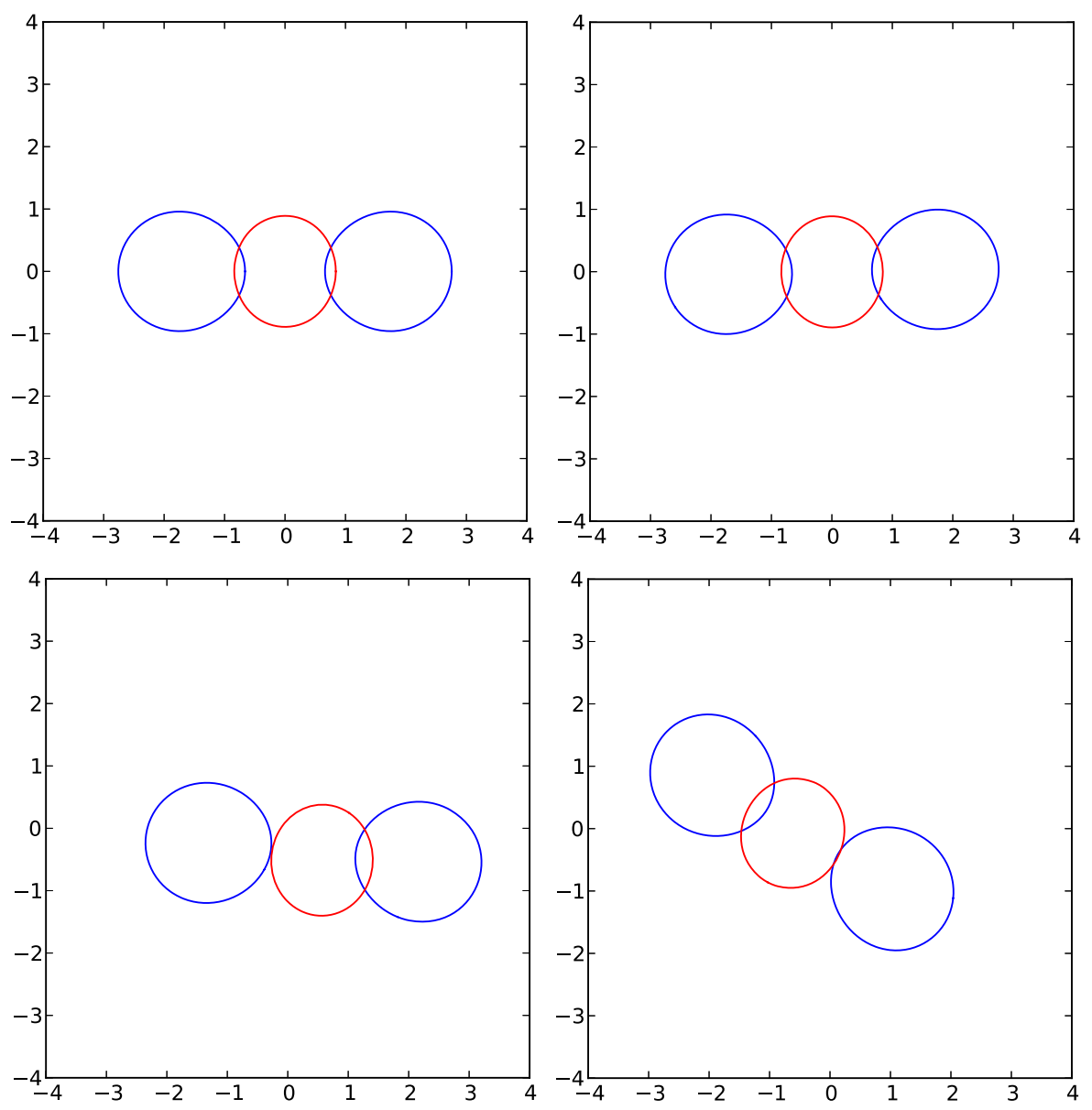

FIG. 15. Top view of the unstable ordinary roundabout at $t=0,249,399$, and 499 for $\rho_{q}=-2, \mu=-1.5, \gamma=0.6$, and $r_{a}=1.72$, see marker $K$ in Figure 13. The frames are plotted in the reference frame rotating with the equilibrium. vortices elongate in the direction joining their centres (a deformation consistent with an azimuthal mode $m=2$ ). At $t=29$ the two inner edges come close to each other and the two vortices start to join by their sharp edges. This is the characteristic of the instability leading to vortex merger in the binary interaction for like-signed vortices. We see that by $t=30$, the two upper poles have merged into a single pole.

Finally, we consider the nonlinear evolution of a symmetric ordinary roundabout in the first regime of instability. We consider a case where the growth rate is near its peak at $r_{a}$ $=1.72$. Results are presented in Figure 15. In this case, the two like-signed vortices are much less deformed due to their weaker mutual interaction. In fact they are too far apart to merge. The state remains very close to its equilibrium configuration. Recall that the growth rate of the instability is small. Eventually the vortices start to misalign and the three vortices depart from their equilibrium position. However, the vortices do not deform significantly though they move relative to each other. The vortices do not undergo interaction which results in their breaking (by shear) or merger, and the structure remains tripolar.

\section{CONCLUSION}

Half of the overall transport of heat, salinity, and other tracers in the oceans is likely to be due to mesoscale structures. $^{75}$ Systems of vortices in mutual equilibrium are of particular interest as they can travel in the fluid over long distances. When these structures are linearly stable, they are able to persist in the flow for long periods of time. These vortex configurations are therefore central to the transport in the oceans. In this paper, we have focused on the existence and on the properties of equilibria for three horizontally aligned vortices in a two-layer fluid. Such configuration may be, for example, formed by the interaction between two propagating pairs of opposite-signed vortices or hetons. 53,74,76 Hetons themselves are often observed on the oceans, as they can be generated by several physical mechanisms, such as a local density perturbation (ice melting), the baroclinic destabilisation of a coastal current, or of a thin and intense oceanic jet.

Notably, configurations of three collinear vortices have been observed in the Bay of Biscay. ${ }^{70-72}$ The present paper has provided an idealised, theoretical framework where the existence and stability of such configurations of three vortices can be studied. In these configurations, two like-signed vortices lie in the first layer and another vortex of opposite sign in the second layer. For these tripoles, there are two branches of steady solutions. One branch consists of symmetric roundabouts, where the vortex of the second layer lies on the axis of rotation of the pair of like-signed vortices. The full structure rotates about this axis. Such branches are referred to as ordinary (or inverse) roundabouts. The second branch consists of threevortex structures whose centre of rotation is eccentric and is referred to as eccentric roundabouts. When the overall strength 
of the vortices vanishes, the centre of rotation is rejected to infinity and the configuration translates. Such vortex structures are known as tritons. The branches of ordinary/inverse and eccentric roundabouts normally intersect at a point where the rotation rate (or translation velocity) of the structure vanishes. Both branches of solution can be characterised by the horizontal distance separating the two like-signed vortices of the first layer, $2 r_{a}$. Since not all distances vary monotonically across all branches, there is no single, universal distance to be considered for the problem. Typically ordinary or inverse roundabouts can exist at equilibrium over larger ranges of this distance. Branches of eccentric roundabouts and triton normally cease to exist for separation distances between the centres of the lower vortices, $2 r_{a}$, less than a threshold. Only symmetric roundabouts can exist for separations less than this threshold.

In the case where the branches of eccentric and ordinary roundabouts intersect, the branch of eccentric roundabouts (respectively, triton) appears to be linearly stable. This can be related to the fact that the turning point of the branch occurs for values of $r_{a}$ too large to trigger instabilities. Instabilities generally occur for the ordinary roundabouts for smaller separation distances between the vortices only. This indicates that most of eccentric roundabouts are generally long lived.

Instabilities have however been observed for eccentric roundabouts and tritons for large values of $\gamma$. For such vortex configurations, one of the upper-layer vortices is closer to the lower-layer vortex than the other. For large values of this parameter, this upper-layer vortex experiences a strain so high that a sharp corner forms on the vortex inner edge; this sustains stationarity. The corner indicates the presence of a stagnation point and the end of the branch of solution. Such strong deformation in the shape of the vortex may trigger instability. In the nonlinear regime, the instability results in the shedding of a PV filament from this inner edge. The structure then stabilises back and persists as a different, meta-stable tripolar structure surrounded by thin filaments. The filaments eventually disappear.

As mentioned before, for values of $r_{a}$ smaller than the range where eccentric roundabouts may exist, the ordinary and inverse roundabouts can be unstable. In the range of parameters studied, the instability results in the merger of the two upper-layer, like-signed vortices. This results in the formation of a metastable hetonic structure which keeps rotating around the same rotation axis. This type of behaviour depends on the value of $\gamma$, and other regimes could be observed (such as the breaking of the opposite-signed vortex $^{74}$ ).

All the results above have been obtained for the case $h_{1}=h_{2}$. The general case with $h_{1} \neq h_{2}$ needs to be studied in detail. However, an early test, represented in Figure 16, shows $r_{b}$ vs $r_{a}$ and the translation velocity $v_{t}$ (for tritons) or the rotation velocity $\Omega_{p}$ for ordinary and inverse roundabouts vs $r_{a}$ for the case $\mu=-2, \gamma=0.6$ and with $h_{1}=0.2, h_{2}=1-h_{1}=0.8$. Results for both $\rho_{q}=-2$ and -4 are presented. In practice, the curves cannot be distinguished visually on the graph as they are almost identical. In these results, the PV of the vortices of the like-signed vortices is kept to $2 \pi$ as before, and their mean radius is also kept to 1 . The $\mathrm{PV}$ of the opposite-signed vortex
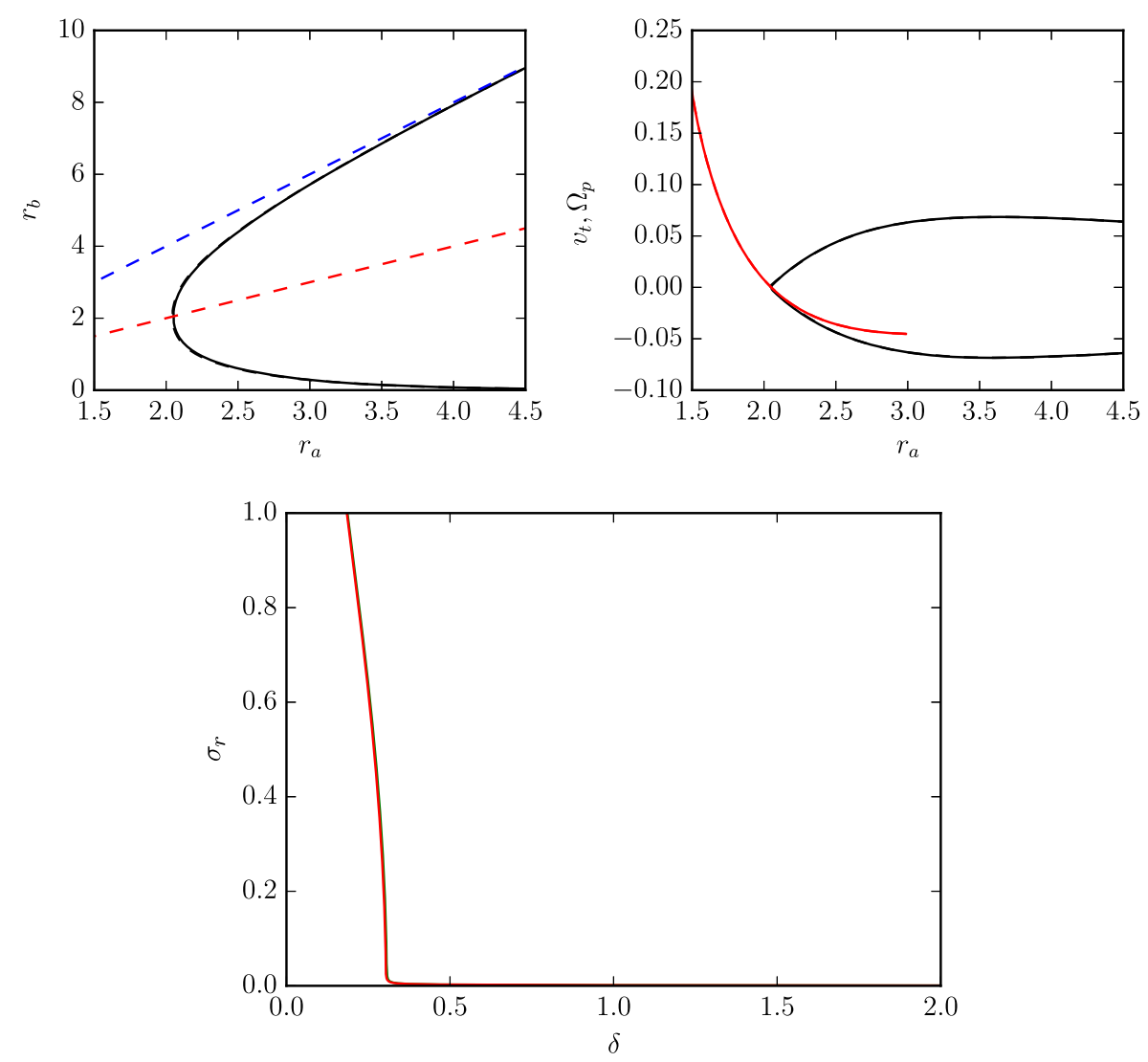

FIG. 16. Steady eccentric and ordinary roundabouts' characteristics for $h_{1}=0.2, h_{2}=0.8$. Here, $\mu=-2$, $\gamma=0.6$. Results for $\rho_{q}=-2$ (solid black-eccentric and solid redordinary/inverse) and $\rho_{q}=-4$ (dashed black-eccentric and dashed redordinary/inverse) are almost superimposed. Top left: distance $r_{b}$ vs $r_{a}$ for the equilibria. Top right: translation velocity (black, eccentric roundabouts) and rotation velocity (red, ordinary/inverse roundabouts) vs $r_{a}$. Bottom: growth rate of the most unstable mode vs the gap $\delta$. 
is then fixed by the value of $\rho_{q}$, and its radius such that the ratio of the volume integrated PV is $\mu$. The linear stability analysis provides results qualitatively similar to those obtained for the case $h_{1}=h_{2}$. The eccentric roundabouts are linearly stable, while ordinary and inverse roundabouts can be unstable when the two like-signed vortices are close enough to each other. We present the growth rate of the most unstable mode for the ordinary roundabout in Figure 16. The overall pattern is the same as the other cases-the difference is in appearance only: here the view is zoomed out to see a larger range of growth rates, and the growth rates are plotted against the distance $\delta$ instead of $r_{a}$. This first plot provides a less detailed view on the growth rates, but allows exhibiting the classical behaviour of the bifurcation of the mode.

The upshot of the study is that, despite the existence of small regions of instability, most of these tripolar structures are linearly stable and are therefore persistent. Such vortex configurations are well suited candidates to be oceanic tracercarrying eddies.

The natural extension of this study is the investigation of the equilibria of four vortices $\left(N_{1}=1, N_{2}=3\right.$, and $N_{1}=N_{2}=2$ ). Point vortex solutions for equilibria have been obtained in the two-layer configuration ${ }^{53,62}$ as well as in the context of the three dimensional, continuously stratified quasi-geostrophic model. ${ }^{57}$ Future work will therefore determine equilibrium solution for finite core vortices and address their linear stability properties.

\section{ACKNOWLEDGMENTS}

M.A.S. and X.C. were supported by RFBR/CNRS (PRC Grant No. 16-55-15001/1069, numerical simulation), and M.A.S. also was supported by RSF (Grant No. 14-50-00095, geophysical applications). The University of St Andrews is a charity registered in Scotland No. SC013532.

\section{APPENDIX: ANALYSIS OF THE DEFORMATION OF VORTEX 3}

We present here a Fourier analysis of the deformation of the boundary of vortex 3 in the case $\mu=-2, \rho_{q}=-4$, and $\gamma=0.8$ when it deforms to form a sharp inner edge. Results are presented in Figure 17. We first measure $\Delta r=r-r_{3}$, the departure of the local polar radius $r$ (measure from centroid of the vortex) as a function of the local polar angle. The contours are originally mapped by 450 nodes. The signal obtained is re-sampled over 512 points equally spaced in polar angle using local quadratic interpolation. We next perform an FFT to determine the azimuthal modes of deformation. The deformation is dominated by mode $m=2$ and its first subharmonics $m=4$. The asymmetry between the inner and outer edges of the vortex is fed by an increase in magnitude of mode $m=3$.
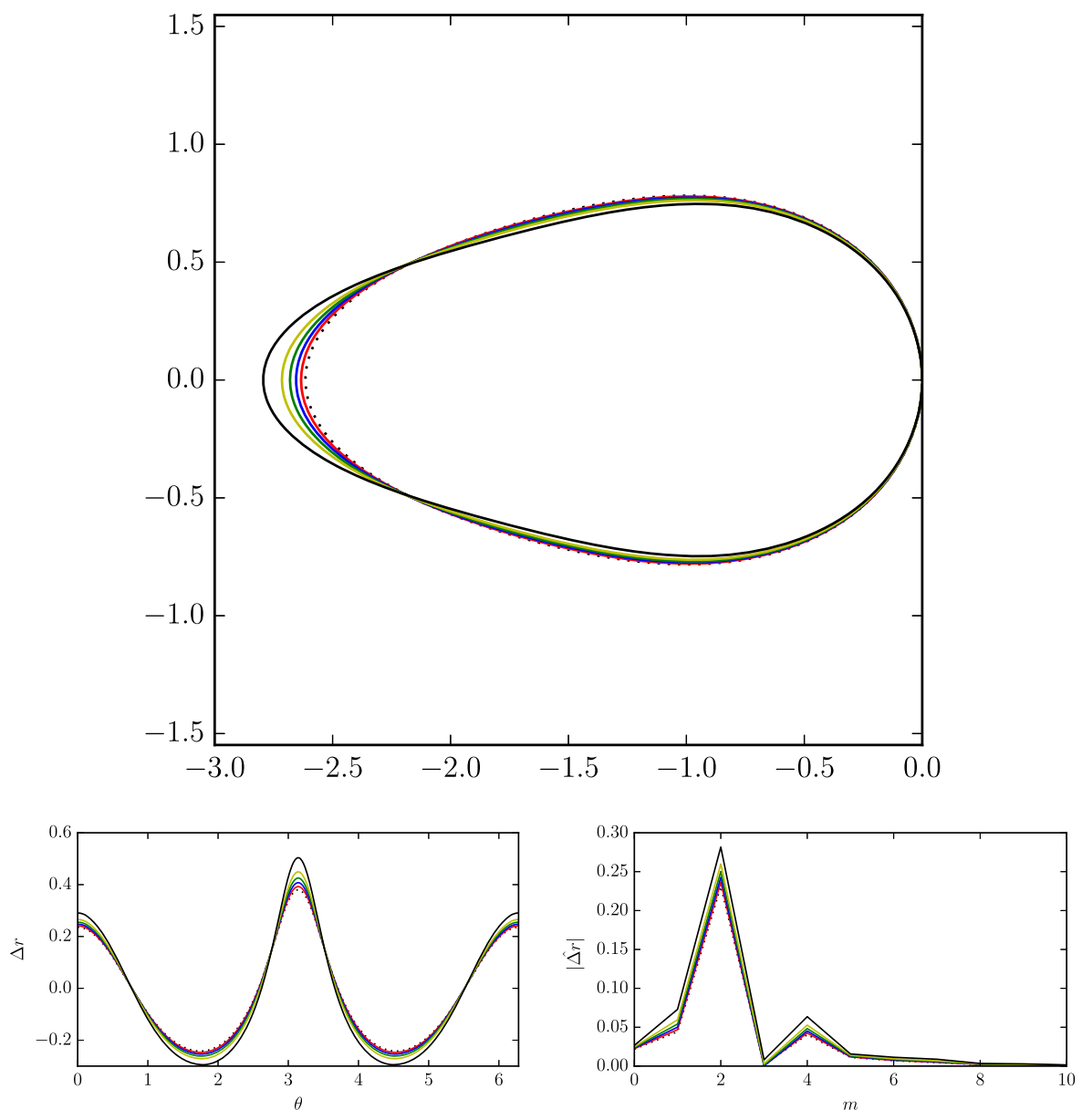

FIG. 17. Shape and deformation of vortex 3 for the eccentric roundabout $\mu=$ $-2, \rho=-4, \gamma=0.8$. Top: boundary of vortex 3 for six steady configurations. All contours are shifted in the $x$-direction such that their outer edge is at the centre of the plane. Bottom left: deformation of the contour $\Delta r$ vs the polar angle $\theta$. Bottom right: norm of the Fourier coefficients of the deformation $|\hat{\Delta r}|$ vs the azimuthal wave number $m$. On the bottom panels: from the least to most deformed the bottom curves are in dotted black, then solid red, solid blue, solid green, solid yellow. The most deformed state obtained numerically corresponds to the solid black line (outer contour on the top panel). 
${ }^{1}$ G. S. Deem and N. J. Zabusky, "Vortex waves: Stationnary 'V-states,' interactions, recurrence, and breaking," Phys. Rev. Lett. 40, 859-862 (1978).

${ }^{2} \mathrm{G}$. Kirchhoff, Vorlesungen über Mathematische Physik: Mechanik (Taubner, Leipzig, 1876).

${ }^{3}$ A. E. H. Love, "On the stability of certain vortex motion," Proc. London Math. Soc. s1-25, 18-43 (1893).

${ }^{4}$ T. B. Mitchell and L. F. Rossi, "The evolution of Kirchhoff elliptic vortices," Phys. Fluids 20, 054103 (2008).

${ }^{5}$ S. A. Chaplygin, "On a pulsating cylindrical vortex," Trans. Phys. Sect. Imperial Moscow Soc. Friends of Nat. Sci. 10, 13-22 (1899) [Regular Chaotic Dyn. 12, 101-116 (1899)]

${ }^{6} \mathrm{~S}$. Kida, "Motion of an elliptic vortex in an uniform shear flow," J. Phys. Soc. Jpn. 50, 3517-3520 (1981).

${ }^{7}$ D. G. Dritschel, "The stability of elliptical vortices in an external straining flow," J. Fluid Mech. 210, 223-261 (1990).

${ }^{8}$ L. M. Polvani and G. R. Flierl, "Generalized Kirchhoff vortices," Phys. Fluids 29, 2376-2379 (1986).

${ }^{9}$ V. F. Kozlov, "Model of two-dimensional vortex motion with an entrainment mechanism," Fluid Dyn. 27, 793-798 (1992).

${ }^{10}$ V. F. Kozlov, "A nonlinear model for Kirchhoff vortex dissipation," Oceanology 32, 427-430 (1992).

${ }^{11}$ V. F. Kozlov, "Model of the interaction of elliptic vortex patches with entrainment effects," Izv., Atmos. Oceanic Phys. 29, 90-96 (1993).

${ }^{12}$ V. S. Sadovskii, "Vortex regions in a potential stream with a jump of Bernoulli's constant at the boundary," J. Appl. Math. Mech. 35, 729-735 (1971).

${ }^{13}$ J. McWilliams, G. R. Flierl, V. D. Larichev, and G. M. Reznik, "Numerical studies of barotropic modons," Dyn. Atmos. Oceans 5, 219-238 (1981).

${ }^{14}$ R. T. Pierrehumbert, "A family of steady, translating vortex pairs with distributed vorticity," J. Fluid Mech. 99, 129-144 (1980).

${ }^{15}$ P. G. Saffman and R. Szeto, "Equilibrium shape of a pair of equal vortices," Phys. Fluids 23, 2339-2342 (1980).

${ }^{16}$ H. M. Wu, E. A. Overman II, and N. J. Zabusky, "Steady-state solutions of the euler equations: Rotating and translating V-states with limiting cases. I. Numerical algorithms and results," J. Comput. Phys. 53, 42-71 (1984).

${ }^{17}$ E. A. Overman II, "Steady-state solutions of the Euler equations in two dimensions. II. Local analysis of limiting V-states," SIAM J. Appl. Math. 46, 765-800 (1986).

${ }^{18}$ Z. Kizner and R. Khvoles, "Two variations on the theme of Lamb-Chaplygin: Supersmooth dipole and rotating multipoles," Regular Chaotic Dyn. 9, 509518 (2004).

${ }^{19}$ A. I. Shavlyugin, "Stationary states of a pair of tangent identical vortex spots in a barotropic ocean," Izv., Atmos. Oceanic Phys. 52, 101-107 (2016).

${ }^{20}$ S. Arendt, "Steadily translating vortices in a stratified fluid," Phys. Fluids 7, 384-388 (1995).

${ }^{21}$ J. Burbea, "On patches of uniform vorticity in a plane of irrotational flow," Arch. Ration. Mech. Anal. 77, 349-358 (1982).

${ }^{22}$ V. V. Perepelkin and A. G. Petrov, "Dynamics of an elliptic vortex," Fluid Dyn. 18, 539-544 (1983).

${ }^{23}$ B. Turkington, "Corotating steady vortex flows with N-fold symmetry," Nonlinear Anal., Theory, Methods Appl. 9, 351-369 (1985).

${ }^{24}$ Y.-H. Wan, "The stability of rotating vortex patches," Commun. Math. Phys. 107, 1-20 (1986).

${ }^{25}$ V. F. Kozlov and V. G. Makarov, "Simulation of the instability of axisymmetric vortices using the contour dynamics method," Fluid Dyn. 20, 28-34 (1985).

${ }^{26}$ J. Nycander, "Steady vortices in plasmas and geophysical flows," Chaos $\mathbf{4}$, 253-268 (1994).

${ }^{27}$ J. Nycander, "Existence and stability of stationary vortices in uniform shear flow," J. Fluid Mech. 287, 119-132 (1995).

${ }^{28}$ N. R. McDonald, "A new translating quasigeostrophic V-state," Eur. J. Mech., B: Fluids 23, 633-644 (2004).

${ }^{29}$ P. Luzzatto-Fegiz and C. H. K. Williamson, "Stability of elliptical vortices from 'imperfect-velocity-impulse' diagrams," Theor. Comput. Fluid Dyn. 24, 181-188 (2010).

${ }^{30}$ P. Luzzatto-Fegiz and C. H. K. Williamson, "An efficient and general numerical method to compute steady uniform vortices," J. Comput. Phys. 230, 6495-6511 (2011).

${ }^{31}$ H. Płotka and D. G. Dritschel, "Quasi-geostrophic shallow-water vortexpatch equilibria and their stability," Geophys. Asrophys. Fluid Dyn. 106, 574-595 (2012).
${ }^{32}$ H. Płotka and D. G. Dritschel, "Quasi-geostrophic shallow-water doublyconnected vortex equilibria and their stability," J. Fluid Mech. 723, 40-68 (2013).

${ }^{33}$ D. G. Dritschel, "The stability and energetics of corotating uniform vortices," J. Fluid Mech. 157, 95-113 (1985).

${ }^{34}$ V. F. Kozlov, "Construction of the stationary states of vortex patches by the method of perturbations," Izv., Atmos. Oceanic Phys. 27, 77-86 (1991).

${ }^{35}$ D. G. Dritschel, "A general theory for two-dimensional vortex interactions," J. Fluid Mech. 293, 269-303 (1995).

${ }^{36}$ D. G. Crowdy, "A class of exact multipolar vortices," Phys. Fluids 11, 2556-2564 (1999).

${ }^{37}$ D. G. Crowdy, "The construction of exact multipolar equilibria of the twodimensional Euler equations," Phys. Fluids 14, 257-267 (2002).

${ }^{38}$ P. Caillol and R. Grimshaw, "Steady multipolar planar vortices with nonlinear critical layer," Geophys. Astrophys. Fluid Dyn. 98, 473-506 (2004).

${ }^{39}$ Z. Kizner and R. Khvoles, "The tripole vortex: Experimental evidence and explicit solutions," Phys. Rev. E 70, 016307 (2004).

${ }^{40}$ Z. Kizner, R. Khvoles, and J. C. McWilliams, "Rotating multipoles on the f- and $\gamma$-planes,” Phys. Fluids 19, 016603 (2007).

${ }^{41}$ Z. I. Kizner, "Rossby solitons with axially symmetric baroclinic modes," Dokl. (Trans.) USSR Acad. Sci. 275, 211-214 (1984).

${ }^{42}$ V. F. Kozlov, V. G. Makarov, and M. A. Sokolovskiy, "Numerical model of the baroclinic instability of axially symmetric eddies in two-layer ocean," Izv., Atmos. Oceanic Phys. 22, 674-678 (1986).

${ }^{43}$ M. A. Sokolovskiy, "Numerical modelling of nonlinear instability for axisymmetric two-layer vortices," Izv., Atmos. Oceanic Phys. 24, 536-542 (1988).

${ }^{44}$ L. M. Polvani, N. J. Zabusky, and G. R. Flierl, "Two-layer geostrophic vortex dynamics: 1. Upper-layer V-states and merger,” J. Fluid Mech. 205, 215-242 (1989).

${ }^{45}$ L. M. Polvani, "Two-layer geostrophic vortex dynamics. 2. Alignment and two-layer V-states," J. Fluid Mech. 225, 241-270 (1991).

${ }^{46}$ Z. I. Kizner, "Solitary Rossby waves with baroclinic modes," J. Mar. Res. 55, 671-685 (1997).

${ }^{47}$ M. A. Sokolovskiy, "Stability of an axisymmetric three-layer vortex," Izv., Atmos. Oceanic Phys. 33, 16-26 (1997).

${ }^{48}$ M. A. Sokolovskiy and J. Verron, "Finite-core hetons: Stability and interactions," J. Fluid Mech. 423, 127-154 (2000).

${ }^{49}$ J. N. Reinaud and D. G. Dritschel, "The merger of vertically offset quasigeostrophic vortices,” J. Fluid Mech. 469, 287-315 (2002).

${ }^{50}$ Z. Kizner, D. Berson, and R. Khvoles, "Baroclinic modon equilibria on the beta-plane: Stability and transitions," J. Fluid Mech. 468, 239-270 (2002).

${ }^{51}$ Z. Kizner, D. Berson, and R. Khvoles, "Non-circular baroclinic modons: Constructing stationary solutions," J. Fluid Mech. 489, 199-228 (2003).

${ }^{52}$ J. N. Reinaud and X. Carton, "The stability and non-linear evolution of quasi-geostrophic hetons," J. Fluid Mech. 636, 109-135 (2009).

${ }^{53}$ M. A. Sokolovskiy and X. Carton, "Baroclinic multipole formation from heton interaction," Fluid Dyn. Res. 42, 045501 (2010).

${ }^{54}$ V. G. Makarov, M. A. Sokolovskiy, and Z. Kizner, "Doubly symmetric finite-core heton equilibria,” J. Fluid Mech. 708, 397-417 (2012).

${ }^{55}$ J. N. Reinaud, "On the stability of continuously stratified quasi-geostrophic hetons,” Fluid Dyn. Res. 47, 035510 (2015).

${ }^{56}$ B. Shteinbuch-Fridman, V. Makarov, X. Carton, and Z. Kizner, "Two-layer geostrophic tripoles comprised by patches of uniform potential vorticity," Phys. Fluids 27, 036602 (2015).

${ }^{57}$ J. N. Reinaud and X. Carton, "The interaction between two oppositely travelling, horizontally offset, antisymmetric quasi-geostrophic hetons," J. Fluid Mech. 794, 409-443 (2016).

${ }^{58}$ X. Carton, D. Ciani, J. Verron, J. Reinaud, and M. Sokolovskiy, "Vortex merger in surface quasi-geostrophy," Geophys. Astrophys. Fluid Dyn 110, $1-22$ (2016)

${ }^{59}$ M. A. Sokolovskiy and J. Verron, "New stationary solutions to the problem of three vortices in a two-layer fluid," Dokl. Phys. 47, 233-237 (2002).

${ }^{60}$ M. A. Sokolovskiy and J. Verron, "Dynamics of the triangular two-layer vortex structures with zero total intensity," Regular Chaotic Dyn. 7, 435-472 (2002).

${ }^{61}$ M. A. Sokolovskiy and J. Verron, "Dynamics of the three vortices in twolayer rotating fluid," Regular Chaotic Dyn. 9, 417-438 (2004).

${ }^{62}$ M. A. Sokolovskiy and J. Verron, Vortex Structures in a Stratified Rotating Fluid (Springer, Switzerland, 2014).

${ }^{63}$ M. A. Sokolovskiy, K. V. Koshel, and J. Verron, "Three-vortex quasigeostrophic dynamics in a two-layer fluid. Part 1. Analysis of relative and absolute motions," J. Fluid Mech. 717, 232-254 (2013). 
${ }^{64}$ K. V. Koshel, M. A. Sokolovskiy, and J. Verron, "Three-vortex quasigeostrophic dynamics in a two-layer fluid. Part 2. Regular and chaotic advection around the perturbed steady states," J. Fluid Mech. 717, 255-280 (2013).

${ }^{65}$ Z. Kizner, "Stability of point-vortex multipoles revisited," Phys. Fluids 23, 064104 (2001).

${ }^{66} \mathrm{Z}$. Kizner, "On the stability of two-layer geostrophic point-vortex multipoles," Phys. Fluids 26, 046602 (2014).

${ }^{67}$ L. G. Kurakin, I. V. Ostrovskaya, and M. A. Sokolovskiy, "Stability of discrete vortex multipoles in homogeneous and two-layer rotating fluid," Dokl. Phys. 66, 217-223 (2015).

${ }^{68}$ L. G. Kurakin, I. V. Ostrovskaya, and M. A. Sokolovskiy, "On the stability of discrete tripole, quadrupole, Thomson' vortex triangle and square in a two-layer/homogeneous rotating fluid," Regular Chaotic Dyn. 21, 291-334 (2016).

${ }^{69}$ B. Shteinbuch-Fridman, V. Makarov, and Z. Kizner, "Transitions and oscillatory regimes in two-layer geostrophic hetons and tripoles," J. Fluid Mech. 810, 535-553 (2017)

${ }^{70}$ R. Pingree and B. Le Cann, "Anticyclonic eddy X91 in the southern Bay of Biscay, may 1991 to february 1992," J. Geophys. Res. 97, 14353-14367, doi:10.1029/92jc01181 (1992).
${ }^{71}$ E. Fernandez, F. Alvarez, R. Anadon, S. Barqueri, A. Bode, A. Garcia, C. Garcia-Soto, J. Gil, N. Gonzalez, A. Iriarte, B. Mourino, F. Rodriguez, R. Sanchez, E. Teira, S. Torres, L. Valdes, M. Valera, R. Valera, and M. Zapata, "The spatial distribution of plankton communities in a slope water anticyclonic oceanic eddy (swoddy) in the southern Bay of Biscay," J. Mar. Biol. Assoc. U.K. 84, 501 (2004).

${ }^{72}$ R. Sanchez and J. Gil, "3D structure, mesoscale interactions and potential vorticity conservation in a swoddy in the Bay of Biscay," J. Mar. Sys. 46, 47-68 (2004).

${ }^{73}$ D. G. Dritschel, "Contour surgery: A topological reconnection scheme for extended integrations using contour dynamics," J. Comput. Phys. 77, 240 266 (1988).

${ }^{74}$ J. N. Reinaud and X. Carton, "Existence, stability and formation of baroclinic tripoles in quasi-geostrophic flows," J. Fluid Mech. 785, 1-30 (2015).

${ }^{75}$ Z. Zhang, W. Wang, and B. Qiu, "Oceanic mass transport by mesoscale eddies," Science 345, 322-324 (2014).

${ }^{76}$ P. L'Hegaret, X. Carton, I. Ambar, C. Menesguen, B. Hua, L. Chérubin, A. Aguiar, B. Le Cann, N. Daniault, and N. Serra, "Evidence of mediterranean water dipole collision in the Gulf of Cadiz," J. Geophys. Res. 119, 5337-5359, doi:10.1002/2014jc009972 (2014). 